\title{
An Empirical Study on the Influencing Factors of Supplier Involvement in New Product Development
}

\author{
(C) Higher Education Press and Springer-Verlag 2010
}

\begin{abstract}
This research identifies six driving factors and twelve enabling factors for supplier involvement in new product development (SINPD) in China via a meta-analysis of the extant literature and a survey of over 100 Chinese manufacturing enterprises. Results show that most suppliers of Chinese manufacturing enterprises engage in new product development at middle or later phase, and the degree of involvement is usually high. Moreover, there are differences in the implementation of SINPD in enterprises of different sizes, types, and industries. The impact of different driving factors and enabling factors on SINPD implementation also varies with enterprise types.
\end{abstract}

Keywords supplier involvement, new product development, driving factors, enabling factors

\section{Introduction}

In an environment of global competition, product life cycle has become

Translated from Keyan Guanli 科研管理 (Science Research Management), 2009, (2): 84-93

Suicheng Li

School of Management, Xi' an University of Technology, Xi'an 710054, China

E-mail: Lisc@xaut.edu.cn

Shanshan Gu $(\bowtie)$

School of Management, Xi' an University of Technology, Xi' an 710054, China

E-mail: gushan6895@126.com

Qiao Wang

Fuzhou Zhuoyuan Culture \& Media Co., Ltd., Fuzhou 350005, China

E-mail: wangqiao_0715@163.com 
increasingly shorter than before. Customers now demand customization and short order-to-delivery cycle. Therefore, new product development has become a key source of competitive advantage.

The uncertainty of technology and environment, however, makes the development of new products increasingly a difficult task for enterprises. Therefore, many enterprises in different industries are now actively engaged in cross-boundary cooperation, such as establishing vertical cooperation with suppliers or developing new products with assistance from outside. For example, Chrysler has long adopted the SINPD system, consisting of new product development, shortened product development cycle and reduced development and manufacturing cost.

SINPD initially appeared in Japan's automobile industry in the 1940s, when a group of engineers in Toyota joined Nipondenso Company in 1949 and initiated the earliest supplier involvement in product research and development stage. This has aroused great interests among Western researchers and practitioners. Imai, Nonaka and Takeuchi (1985) first explored supplier's involvement in early stage of product development and its positive impact (Bidault, Despres and Bulter, 1998). A few years later, Clark and Fujimoto (1991) confirmed the importance of SINPD empirically. More research on SINPD appeared in the 1990s. A majority of them focused on the drivers of SINPD (Bidault, Despres and Bulter, 1998), SINPD under different cultural backgrounds (Song and Parry, 1999), and the role of SINPD in the development cycle and flexibility (Lamming, 1993).

In the $21^{\text {st }}$ century, researchers have become concerned about the influencing factors of SINPD (Walter, 2003). SINPD, while enhancing firm performance, has also made management more complicated. There are many factors which can make or break SINPD, such as conflict of management and mutual coordination (Fliess and Becker, 2006). The influencing factors of SINPD are divided into driving factors and enabling factors. However, most of the extant studies on the influencing factors of SINPD are based on case study or literature analysis, while empirical research is lacking.

In recent years, a growing number of Chinese companies have started to implement SINPD. However, in order to implement SINPD more effectively, they must identify the influencing factors of SINPD first. Due to differences in economic, political, and cultural environments, the influencing factors for SINPD in Chinese enterprises might be different from factors identified in foreign literature. The situation might be the same for driving and enabling factors of SINPD (Fliess and Becker, 2006; McIvor and Humphreys, 2004; Petersen, Handfield and Ragatz, 2003; Petersen, Handfield and Ragatz, 2005; Wagner and Hoegl, 2006; Walter, 2003). This paper attempts to identify and confirm the driving and enabling factors of SINPD in China. It also explores the different 
implementation of SINPD in various types of companies, and further studies the impact of influencing factors on SINPD implementation. It aims to enrich the theory of SINPD in the Chinese culture and guide Chinese manufacturing enterprises to implement SINPD more effectively.

This paper is organized as follows: Section 2 is a literature review on the influencing factors of SINPD. Section 3 explores the influencing factors of SINPD in Chinese enterprises. Section 4 builds a theoretical model of the relationship between influencing factors of SINPD and implementation of SINPD, and evaluates the model using regression analysis. Managerial suggestions and implications are provided in the last section.

\section{Literature Review}

\subsection{Driving Factors}

A driving factor deals with a need for SINPD, which explains why companies desire to implement SINPD. Sako (1992) pointed out that there are many factors behind a manufacturer's implementation of SINPD, including national and cultural factors, industry characteristics (e.g., asset specificity) and enterprise goals (Clark, 1989). Fujimoto (1994) analyzed the factors that affect the implementation of SINPD, and divided these factors into three categories: First, environmental pressures included shortened development time, technology integration, product complexity, component reliability and the industrial sector. Second, social and industrial criteria referred to geographical origin, the scope for competition, industry characteristics. Third, organizational choice captures the degree of integration, procurement rate and the supplier activities (Birou and Fawcett, 1994).

In addition, many researchers studied firms' business environment and its influences on organizational structure and strategy (Clark and Fujimoto, 1991). They confirmed that the driving factors of SINPD from three levels, namely business units, project-level relations, and cooperation (see Table 1).

Business Unit Level. Driving factors of SINPD at the business unit level mainly include market and technology uncertainty (Birou and Fawcett, 1994; Eisenhardt and Tabrizi, 1995; Fine, 2000; Clark and Fujimoto, 1991; Dobler and Burt, 1996; Dowlatshahi, 1998), R\&D dependence, supplier dependence, company size, and product flexibility (Wynstra et al., 2000; Dowlatshahi, 1992).

Project Level. Factors at the project level mainly refer to the innovation objectives of a project. Prior studies show that the driving factors of SINPD depend on project characteristics (Griffin and Page, 1996; Duarte and Davies, 2003). Researchers have also found that a project's innovation objectives and its importance depend on the actual levels of innovation (Griffin and Page, 1996; 
Tatikonda and Rosenthal, 2000; Duarte and Davies, 2003; Dyer and Ouchi, 1993).

Cooperative Relationship Level. Influencing factors at the cooperative relationship level include complexity of component development, uncertainty of component technology, the role of components in the entire system and the level of supplier competitiveness (Wasti and Liker, 1997; Wynstra and Pierick, 2000; Dowlatshahi, 1992; Eisenhardt and Tabrizi, 1995).

Table 1 Potential Driving Factors of SINPD

\begin{tabular}{|c|c|c|c|}
\hline Level & Study & Variable & Driving factor \\
\hline \multirow{4}{*}{$\begin{array}{l}\text { Business unit } \\
\text { level }\end{array}$} & $\begin{array}{l}\text { Eisenhardt and } \\
\text { Tabrizi, } 1995\end{array}$ & $\begin{array}{l}\text { The effectiveness of } \\
\text { experience-based } \\
\text { retrenchment strategy }\end{array}$ & $\begin{array}{l}\text { Technological uncertainties; } \\
\text { unpredictability of the } \\
\text { project/environmental } \\
\text { uncertainty }\end{array}$ \\
\hline & Fine, 2000 & $\begin{array}{l}\text { The effectiveness of } \\
\text { outsourcing }\end{array}$ & $\begin{array}{l}\text { Industrial difference in } \\
\text { technological and market } \\
\text { uncertainty }\end{array}$ \\
\hline & $\begin{array}{l}\text { Birou and Fawcett, } \\
1994\end{array}$ & $\begin{array}{l}\text { The role of supplier } \\
\text { involvement }\end{array}$ & $\begin{array}{l}\text { Competition conditions in the } \\
\text { manufacturer market }\end{array}$ \\
\hline & $\begin{array}{l}\text { Wynstra et al., } \\
2000\end{array}$ & $\begin{array}{l}\text { The necessity of } \\
\text { supplier involvement }\end{array}$ & $\begin{array}{l}\text { R\&D dependence; supplier } \\
\text { dependence; enterprise size; } \\
\text { product complexity }\end{array}$ \\
\hline \multirow{2}{*}{ Project level } & $\begin{array}{l}\text { Griffin and Page, } \\
1996\end{array}$ & $\begin{array}{l}\text { Project performance } \\
\text { measurement }\end{array}$ & Product innovation degree \\
\hline & $\begin{array}{l}\text { Tatikonda and } \\
\text { Rosenthal, } 2000\end{array}$ & $\begin{array}{l}\text { Results of project } \\
\text { implementation }\end{array}$ & $\begin{array}{l}\text { Technology novelty; project } \\
\text { complexity }\end{array}$ \\
\hline \multirow{2}{*}{$\begin{array}{l}\text { Cooperative } \\
\text { relationship } \\
\text { level }\end{array}$} & $\begin{array}{l}\text { Wasti and Liker, } \\
1997\end{array}$ & $\begin{array}{l}\text { Degree of supplier } \\
\text { involvement }\end{array}$ & $\begin{array}{l}\text { Technology uncertainty of } \\
\text { component elements; } \\
\text { supplier market } \\
\text { competitiveness }\end{array}$ \\
\hline & $\begin{array}{l}\text { Wynstra and } \\
\text { Pierick, } 2000\end{array}$ & $\begin{array}{l}\text { Differentiated } \\
\text { management of the } \\
\text { suppliers involved }\end{array}$ & $\begin{array}{l}\text { Development risk; } \\
\text { involvement degree }\end{array}$ \\
\hline
\end{tabular}

\subsection{Enabling Factors}

Enabling factors affect organizational capacity when implementing certain projects (Wynstra et al., 2003). In contrast to driving factors, they facilitate an organization to conduct certain activities under specific scenarios (Van Echtelt and Wynstra, 2001). Many researchers have studied potential factors promoting SINPD management, including internal factors of manufacturers, external factors (i.e., the characteristics of suppliers), cooperative relationship between manufacturers and suppliers (see Table 2). 
Table 2 Potential Enabling Factors of SINPD

\begin{tabular}{|c|c|c|}
\hline Level & Study & Enabling factor \\
\hline \multirow{3}{*}{ Internal factors } & $\begin{array}{l}\text { Wynstra et al., 2000; Burt and Soukup, } \\
1985\end{array}$ & $\begin{array}{l}\text { Internal organization of the } \\
\text { procurement department } \\
\text { and } R \& D \text { team }\end{array}$ \\
\hline & Wynstra et al., 2000 & $\begin{array}{l}\text { Information archiving and } \\
\text { exchange }\end{array}$ \\
\hline & $\begin{array}{l}\text { Anklesaria and Burt, 1987; Guy and Date, } \\
\text { 1993; Atuahene-Gima, 1995; Dobler and } \\
\text { Burt, } 1996\end{array}$ & Human resources quality \\
\hline External factors & $\begin{array}{l}\text { Waisti and Liker, 1997; Hartlet and Zirger, } \\
\text { 1997; Birou and Fawcett, 1994; Handfiled } \\
\text { et al., } 1999\end{array}$ & $\begin{array}{l}\text { Supplier's ability of } \\
\text { technological innovation }\end{array}$ \\
\hline \multirow{3}{*}{$\begin{array}{l}\text { Cooperative } \\
\text { relationship } \\
\text { factors }\end{array}$} & $\begin{array}{l}\text { Waisti and Liker, 1997; Farr and Fisher, } \\
1992\end{array}$ & Experience of cooperation \\
\hline & $\begin{array}{l}\text { Lorange, 1988; Perlmutter and Heenan, } \\
\text { 1986; Whipple and Frankel, 2000; Bruce } \\
\text { et al., } 1995\end{array}$ & $\begin{array}{l}\text { Consistency between culture } \\
\text { and operation }\end{array}$ \\
\hline & $\begin{array}{l}\text { Sako, 1992; Gabarro, 1987; Bensaou } \\
\text { Michael, } 2000\end{array}$ & Trust \\
\hline
\end{tabular}

Furthermore, an additional 12 important factors were found which can influence SINPD performance (Ragatz, Handheld and Scannell, 1997), including supplier involvement in a manufacturer's project team, direct inter-company and cross-functional communication, share of education and training, and senior manager commitment. The importance is stressed of senior manager commitment, cultural compatibility and awareness of external environment trends to SINPD performance (Bruce et al., 1995). Based on a questionnaire survey, Littler et al. (1995) found that the formation of mutual-shared principles, objectives, tasks, and frequent communication are important to SINPD.

Recently, some researchers have claimed that it is difficult to reach consensus on results in SINPD research (Primo and Amundson, 2002; Wagner, 2006; Wynstra et al., 2001). However, researchers have proposed that good management at the following two levels is essential to the successful implementation of SINPD:

Organizational Level. Organizational level management is twofold: First, from the technical perspective, the product system, design types and development mode of both the manufacturer and supplier should match with each other (Boutellier and Wagner, 2003). More cooperation shall be pursued among different suppliers and between suppliers and manufacturers (e.g., all parties involved can participate in the development process) (Von Hippel, 1990); Second, from the R\&D capability perspective, considering the complexity nature 
of new product development, only suppliers with adequate R\&D capabilities shall be invited to participate in SINPD.

Project Level. SINPD at project level focuses on the relationship between manufacturers and suppliers, especially the mutual interaction among project team members from both the manufacturer and supplier. Empirical research has shown that in SINPD projects, it is important to promote the cooperation quality between manufacturer and supplier. However, improvement in degree of mutual sharing between the two partners is not that important (Hoegl and Wagner, 2005).

As shown above, prior studies on influencing factors of SINPD are mainly conducted from the perspectives of driving and enabling factors, neglecting other possible influencing factors. To identify influencing factors of SINPD in a systematic way, this paper aims to explore the issue from the following aspects:

(1) Confirm the influencing factors of SINPD, including driving and enabling factors in the context of Chinese enterprises;

(2) Further explore the differences in implementation of SINPD in different types of Chinese enterprises;

(3) Further analyze the differences in degree of impact of different influencing factors on SINPD implementation and construct a model of influencing factors for SINPD accordingly.

\section{Method}

Steps taken in this paper are listed as follows: First, we identified a list of possible influencing factors of SINPD based on the relevant literature and a multiple case study. We then compiled a preliminary questionnaire accordingly. Based on data collected from Chinese enterprises, we conducted exploratory and confirmatory factor analysis to confirm the hypothesized influencing factors.

Based on the results of factor analysis, we established a model of influencing factors for SINPD. Moderating variables and corresponding hypotheses were introduced to further explore the conditioned degree of influence of supplier involvement on new product development. In the end, we employed regression analysis to test all the hypotheses using the data collected from Chinese enterprises.

A large-scale survey was conducted. The pilot questionnaire mainly included basic personal information, company information, implementation situation of SINPD, driving and enabling factors. Basic personal information includes education level, work experience, department and job title. Company information includes company size, business nature and industry type. The implementations of SINPD mainly refers to the involvement time (IVT) and degree (IVD). The questionnaire consisted of 31 items for driving factors and 35 items for enabling 
factors. Except personal information and company information, all questions used 7-point Likert scale. The final questionnaire was compiled based on adjustment of some of the items in the pilot questionnaire and was sent to sampled enterprises nationwide.

\section{Factor Analysis of SINPD Influencing Factors}

\subsection{Meta-Analysis of SINPD Influencing Factors}

Meta-analysis is based on re-survey, re-analysis and synthesis of the extant literature (Ragatz, Handheld and Scannell, 1997). A survey of the extant literature on the driving factor of SINPD identified 101 driving factors out of 32 relevant research papers, and 106 enabling factor out of 43 papers. To guarantee the validity of factors extracted, we eliminated some repeated calculated factors, resulting in 13 driving factors and 15 enabling factors. The meta-analysis of the driving and enabling factors for SINPD are listed in Fig. 1 and Fig. 2, as below.

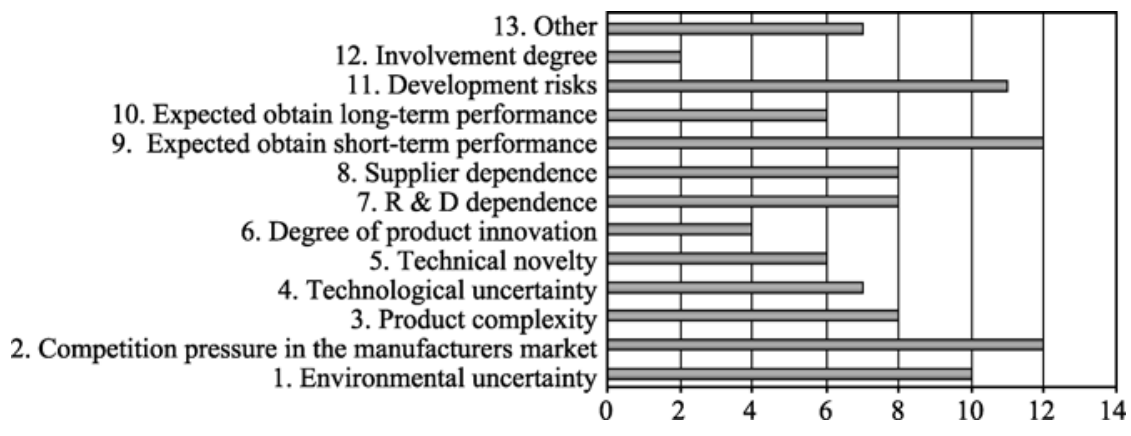

Fig. 1 Meta-Analysis of Driving Factors of SINPD

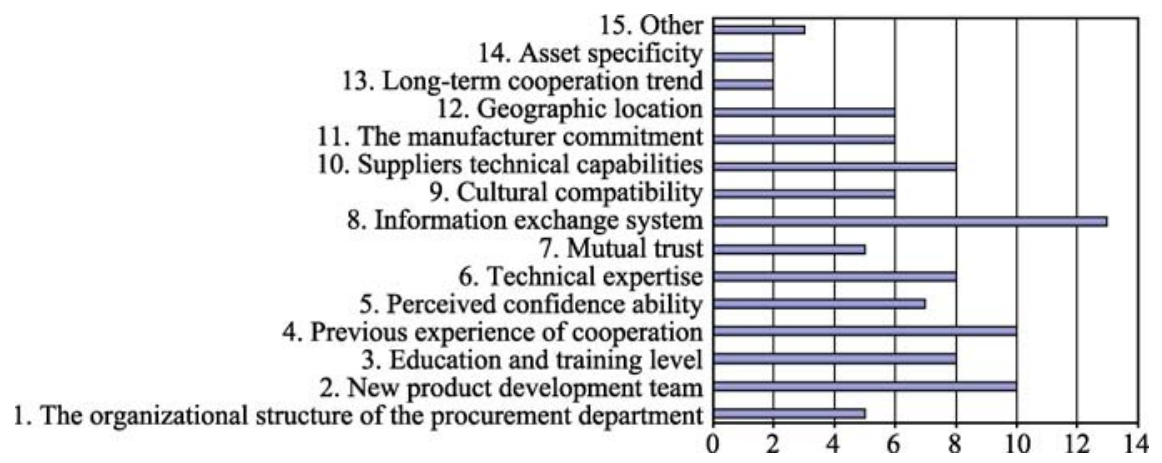

Fig. 2 Meta-Analysis of Enabling Factors of SINPD 
As shown in Fig. 1, involvement degree and product innovation degree are less representative than other factors, and thus can be eliminated from further analysis. The 10 remaining driving factors are: environmental uncertainty, competition pressure in the manufacturers market, product complexity, technological uncertainty, technical novelty, R\&D dependence, supplier dependence, expected obtain short-term performance, expected obtain long-term performance and development risks. These factors, however, need to be further validated by empirical study based on data from Chinese manufacturing industry to help people gain a better understanding of SINPD in China.

Similarly, in Fig. 2, the asset-specific, long-term cooperation tendency is less representative than other factors and thus can be eliminated from the final factors. Therefore, the 12 final enabling factors are: the organizational structure of the procurement department, new product development team, education and training level, previous experience of cooperation, perceived confidence ability, technical expertise, mutual trust, information exchange system, cultural compatibility, suppliers technical capabilities, the manufacturer commitment and geographic location.

\subsection{Exploratory Interviews}

Exploratory interviews were conducted in Shaanxi Auto Group, Xi'an High Voltage Switchgear Electric Co., Ltd. and Xi' an Datang Telecom Co., Ltd. All of the three enterprises had implemented SINPD in the previous years. Descriptive statistics are shown in Table 3.

The participants were mainly SINPD involved technical managers in the R\&D department, directors of purchasing department, and senior managements. Participants aged between 35 and 45, and obtained at least college education, with 10-plus years of total work experience and 5-plus years' work at present company.

Table 3 Descriptive Statistics of Interviewees and Sampled Enterprises

\begin{tabular}{|c|c|c|c|c|c|c|}
\hline Company & $\begin{array}{l}\text { Year of } \\
\text { establishment }\end{array}$ & Industry & $\begin{array}{r}\text { Total } \\
\text { assets } \\
\text { (billion } \\
\text { Yuan) }\end{array}$ & $\begin{array}{l}\text { No. of } \\
\text { employees }\end{array}$ & $\begin{array}{l}\text { No. of } \\
\text { employees } \\
\text { in product } \\
\text { development }\end{array}$ & $\begin{array}{l}\text { No. of } \\
\text { employees } \\
\text { surveyed }\end{array}$ \\
\hline $\begin{array}{l}\text { Shaanxi } \\
\text { Auto } \\
\text { Group }\end{array}$ & 1968 & $\begin{array}{l}\text { Heavy-duty } \\
\text { trucks }\end{array}$ & 1.98 & 11000 & 2000 & 6 \\
\hline $\begin{array}{l}\text { Xi'an High } \\
\text { Voltage } \\
\text { Switchgear } \\
\text { Electric }\end{array}$ & 1955 & $\begin{array}{l}\text { Electrical } \\
\text { equipment }\end{array}$ & 2.8 & 6302 & 1208 & 4 \\
\hline $\begin{array}{l}\text { Xi'an Datang } \\
\text { Telecom }\end{array}$ & 1993 & $\begin{array}{l}\text { Electronic } \\
\text { equipment }\end{array}$ & 3.76 & 4000 & 2300 & 5 \\
\hline
\end{tabular}


The main purpose of the interview was to confirm the driving and enabling factors in these enterprises. Semi-structured interviews were conducted, and recorded for analysis afterwards.

Our interview results showed that most of the driving factors of SINPD in Chinese enterprises were consistent with that of found by Western researchers. Meanwhile, we also found two new factors, namely national innovation policy guidance and technical capacity of suppliers. Including the above mentioned 10 driving factors, we find 12 driving factors of SINPD in Chinese enterprises.

We also found two new enabling factors for SINPD of Chinese enterprises, namely mutual attraction and the organizational structure of $R \& D$ department. The 12 enabling factors for Chinese enterprises, which can be divided into four different dimensions: organizational management, cooperation relationship, human resources quality, and mutual attraction, as shown in Fig. 3.

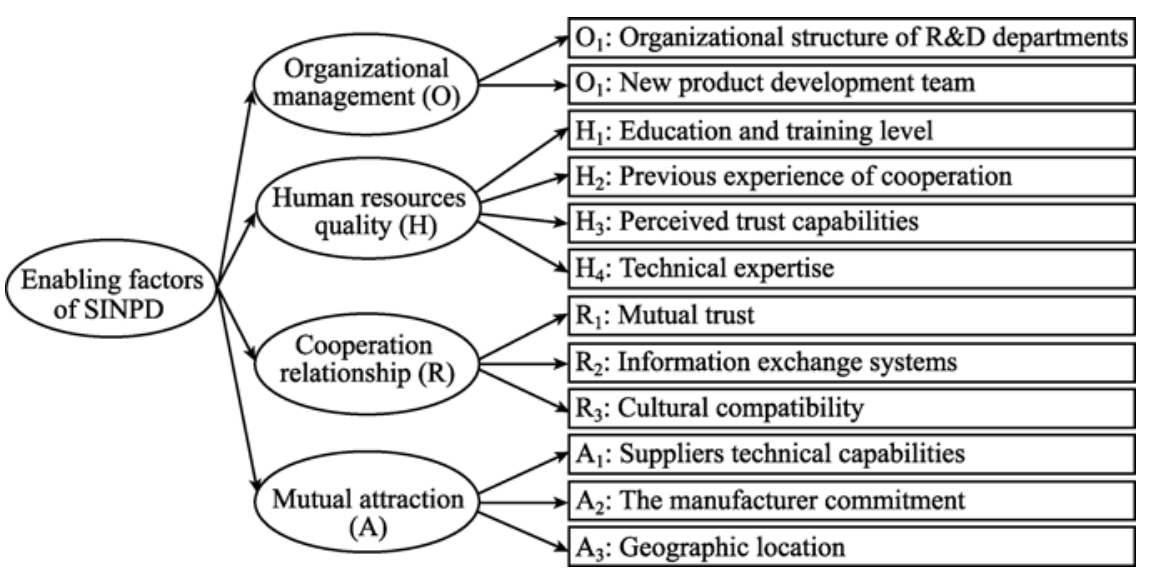

Fig. 3 Factor Model of Enabling Factors

\subsection{The Survey}

Questionnaires were delivered to more than 40 companies with SINPD in seven different provinces in China (i.e., Shaanxi, Shanxi, Ningxia, Shandong, Zhejiang, Jiangsu, and Jilin). In total, 162 questionnaires were returned out of the 200 questionnaires distributed (return rate $=81 \%$ ), including 149 valid ones (valid return rate $=74.5 \%$ ).

Data collected are representative of the SINPD-implemented enterprises, since the sampled enterprises are widely distributed in a number of different industries and questionnaires were filled in by employees from $R \& D$ (or R\&D-related) departments. These data are therefore suitable for analysis in the next step. 


\subsection{Analysis of Driving Factors}

In light of the lack of consensus on the driving factors of SINPD, we try to identify the key driving factors for SINPD by using exploratory factor analysis method. SPSS11.5 software package is used for analysis.

Reliability test is mainly used to test the internal consistency of a scale. The results of reliability test of the 149 valid questionnaires show that the Cronbach's $\alpha$ of the 12 scales are $0.7749,0.9456,0.8398,0.7405,0.8140,0.7939$, $0.9134,0.7089,0.9046,0.9383,0.7575,0.786$ 7, respectively. As all the Cronbach's $\alpha$ are greater than 0.7 , the scales used have satisfactory reliability.

As a rule, before conducting factor extraction, we need to do an item analysis to determine the critical ratio (CR) for each questionnaire item. According to the participants' response to each item, we divided questionnaire data into a high group and a low group. We then used independent sample $t$-test to test the differences between this two groups for each item and deleted all items that are not significant (at 0.05 level) in $t$-test.

Principal factor analysis (PFA) was used for factor extraction by selecting the common factors with eigenvalue above 1.0 and then rotate them using greatest variance method. Items were kept with community larger than 0.6 and factor loading larger than 0.5. According to Kaiser (1974), the closer the KMO value to 1 , the more suitable an item is for factor analysis. The KMO value of our 38 items is 0.875 , showing that these items are suitable for factor analysis. In addition, their approximate chi-square $\left(\chi^{2}\right)$ value is 18429.388 , showing that there exist common factors. Table 4 shows that there are eight common factors, and the cumulative explained variance amounts to $74.777 \%$.

Table 4 Total Variance Explained

\begin{tabular}{|c|c|c|c|c|c|c|c|c|c|}
\hline \multirow{2}{*}{ Factor } & \multicolumn{3}{|c|}{ Initial eigenvalue } & \multicolumn{3}{|c|}{$\begin{array}{l}\text { Extraction sum of squared } \\
\text { loadings }\end{array}$} & \multicolumn{3}{|c|}{$\begin{array}{l}\text { Rotation sum of squared } \\
\text { loadings }\end{array}$} \\
\hline & Sum & $\begin{array}{l}\% \text { of } \\
\text { variance }\end{array}$ & $\begin{array}{l}\text { Cumulative } \\
\%\end{array}$ & Sum & $\begin{array}{l}\% \text { of } \\
\text { variance }\end{array}$ & $\begin{array}{l}\text { Cumulative } \\
\%\end{array}$ & Sum & $\begin{array}{l}\% \text { of } \\
\text { variance }\end{array}$ & $\begin{array}{c}\text { Cumulative } \\
\%\end{array}$ \\
\hline 1 & 13.631 & 35.872 & 35.872 & 13.631 & 35.872 & 35.872 & 6.648 & 17.494 & 17.494 \\
\hline 2 & 3.794 & 9.985 & 45.857 & 3.794 & 9.985 & 45.857 & 5.495 & 14.460 & 31.954 \\
\hline 3 & 3.017 & 7.940 & 53.797 & 3.017 & 7.940 & 53.797 & 3.734 & 9.827 & 41.781 \\
\hline 4 & 2.287 & 6.019 & 59.816 & 2.287 & 6.019 & 59.816 & 3.125 & 8.225 & 50.006 \\
\hline 5 & 1.814 & 4.774 & 64.590 & 1.814 & 4.774 & 64.590 & 2.852 & 7.506 & 57.512 \\
\hline 6 & 1.415 & 3.724 & 68.314 & 1.415 & 3.724 & 68.314 & 2.343 & 6.166 & 63.678 \\
\hline 7 & 1.255 & 3.302 & 71.616 & 1.255 & 3.302 & 71.616 & 2.255 & 5.934 & 69.612 \\
\hline 8 & 1.201 & 3.162 & 74.777 & 1.201 & 3.162 & 74.777 & 1.963 & 5.165 & 74.777 \\
\hline
\end{tabular}

Table 5 is the rotated component matrix, which shows the results of 
orthogonal rotation and common degree of every item. Based on the item select standard, which defines common degree less than 0.6 and factor loading less than 0.5 should be deleted, most researchers suggest that one factor must have more than 3 items, otherwise the number of item is too small to detect the representative character of this factor and its content validity would be less rigorous. So after the first factor analysis, item B1, B2, B9, B25, B26 are deleted. As shown in Table 5, the common degree of those remaining items are all bigger than 0.6 , and the volume of each factor loading is bigger than 0.5 .

Table 5 Rotated Component Matrix

\begin{tabular}{|c|c|c|c|c|c|c|c|c|c|}
\hline \multirow[t]{2}{*}{ Item } & \multicolumn{8}{|c|}{ Component } & \multirow{2}{*}{$\begin{array}{c}\text { Common } \\
\text { degree }\end{array}$} \\
\hline & 1 & 2 & 3 & 4 & 5 & 6 & 7 & 8 & \\
\hline B34 & 0.824 & 0.156 & & & 0.158 & 0.195 & 0.104 & & 0.785 \\
\hline B32 & 0.815 & 0.160 & 0.124 & 0.199 & 0.137 & 0.133 & & & 0.785 \\
\hline B33 & 0.811 & 0.199 & 0.114 & 0.127 & 0.126 & 0.128 & & & 0.763 \\
\hline B35 & 0.780 & 0.153 & 0.116 & & 0.111 & 0.235 & & & 0.733 \\
\hline B31 & 0.765 & 0.199 & 0.120 & 0.240 & 0.120 & 0.123 & & & 0.726 \\
\hline B27 & 0.765 & 0.237 & 0.158 & & & & 0.147 & & 0.698 \\
\hline B28 & 0.759 & 0.191 & & & & & & 0.172 & 0.673 \\
\hline B29 & 0.750 & 0.167 & 0.160 & & 0.113 & 0.145 & 0.171 & 0.163 & 0.706 \\
\hline B30 & 0.726 & 0.196 & 0.165 & & 0.151 & & 0.138 & 0.151 & 0.666 \\
\hline B10 & 0.278 & 0.840 & & 0.108 & 0.140 & 0.112 & 0.102 & & 0.848 \\
\hline B11 & 0.235 & 0.834 & 0.124 & 0.128 & & & & 0.173 & 0.826 \\
\hline B13 & 0.222 & 0.831 & 0.114 & 0.122 & & & & 0.182 & 0.814 \\
\hline B14 & 0.293 & 0.826 & & 0.108 & 0.107 & 0.114 & & & 0.825 \\
\hline B7 & 0.113 & 0.806 & & & 0.172 & 0.116 & 0.100 & -0.163 & 0.750 \\
\hline B8 & 0.236 & 0.798 & & & & & & 0.167 & 0.752 \\
\hline B12 & 0.135 & 0.616 & & 0.184 & & & 0.102 & 0.554 & 0.760 \\
\hline B9 & 0.159 & 0.488 & & 0.173 & -0.102 & & & 0.574 & 0.551 \\
\hline B6 & 0.140 & & 0.917 & & 0.194 & & & & 0.908 \\
\hline B5 & 0.170 & & 0.875 & & 0.202 & & & & 0.844 \\
\hline B4 & 0.116 & & 0.873 & & 0.166 & & & & 0.823 \\
\hline B3 & 0.222 & & 0.588 & & 0.191 & 0.112 & 0.112 & 0.394 & 0.619 \\
\hline B1 & 0.230 & 0.192 & 0.478 & 0.174 & & & 0.196 & 0.275 & 0.469 \\
\hline B2 & 0.228 & 0.131 & 0.425 & 0.299 & 0.166 & & & 0.420 & 0.546 \\
\hline B20 & 0.149 & 0.176 & & 0.802 & 0.203 & & & 0.265 & 0.809 \\
\hline B23 & 0.136 & 0.190 & & 0.793 & 0.202 & & & 0.233 & 0.780 \\
\hline
\end{tabular}




\begin{tabular}{|c|c|c|c|c|c|c|c|c|c|}
\hline \multirow{2}{*}{ Item } & \multicolumn{8}{|c|}{ Component } & \multirow{2}{*}{$\begin{array}{c}\begin{array}{c}\text { Common } \\
\text { degree }\end{array} \\
\text { Extraction }\end{array}$} \\
\hline & 1 & 2 & 3 & 4 & 5 & 6 & 7 & 8 & \\
\hline B19 & 0.182 & 0.168 & 0.163 & 0.774 & & & 0.343 & & 0.808 \\
\hline B22 & 0.173 & 0.163 & 0.146 & 0.766 & & & 0.364 & & 0.799 \\
\hline B17 & 0.206 & 0.104 & 0.253 & 0.172 & 0.839 & 0.138 & & & 0.878 \\
\hline B15 & 0.207 & & 0.227 & 0.207 & 0.789 & 0.110 & & 0.111 & 0.799 \\
\hline B16 & 0.227 & & 0.385 & & 0.702 & 0.142 & & & 0.734 \\
\hline B24 & 0.238 & & 0.250 & & 0.564 & & 0.347 & 0.344 & 0.688 \\
\hline B37 & 0.247 & 0.115 & & & & 0.866 & & & 0.832 \\
\hline B36 & 0.185 & & 0.130 & & 0.111 & 0.817 & & & 0.740 \\
\hline B38 & 0.245 & 0.105 & & & 0.138 & 0.774 & 0.103 & & 0.717 \\
\hline B18 & 0.209 & 0.201 & & 0.227 & 0.140 & & 0.826 & & 0.857 \\
\hline B21 & 0.220 & 0.183 & & 0.246 & & & 0.824 & & 0.845 \\
\hline B26 & 0.226 & 0.259 & 0.106 & & 0.476 & & 0.492 & 0.118 & 0.617 \\
\hline B25 & & & & 0.252 & 0.238 & & 0.109 & 0.627 & 0.543 \\
\hline
\end{tabular}

Based on the above selected standards, seven factors are extracted from the remaining items. However, Factor Seven contains only two items, so we eliminated B18 and B21. When conducting the third factor analysis, all items are in line with the standards. Experts were then invited to retest the above analysis and adjusted the retained items. In the end, 31 items were retained for the driving factors questionnaire. Considering there are still too many items for one questionnaire, we use the slip map as a reference standard to extract 6 final factors, which is consistent with the extraction result of using the Eigenvalue greater than 1.0 as a standard. The cumulative variance explained by the six factors reaches $77 \%$, indicating that the structure of the questionnaire has a high validity. Therefore, the six factors are key driving factors of SINPD. Table 6 shows the rotated factor loading matrix, the common degree of each item, Cronbach's $\alpha$ of each dimensions and the correlation coefficient (corrected item-total correlation, CITC) of each item.

As shown in Table 6, the values of CITC are all greater than 0.5 , the Cronbach's $\alpha$ of six sub-scales are all above 0.70, and the total table Cronbach's $\alpha$ value is 0.9428 , which means that the reliability of this scale is satisfactory and the reliability of using those operative variables to measure the corresponding nominal variables is acceptable. We name the final six extracted key driving factors of SINPD as follows: external competitive pressure, national innovation policy guidance, product complexity, technology integration, R\&D dependence, supplier dependence, expected performance. 
Table 6 Rotated Component Matrix, Cronbach's $\alpha$ and Corrected Item-Total Correlation

\begin{tabular}{|c|c|c|c|c|c|c|c|c|c|c|}
\hline \multirow{2}{*}{ Item } & \multirow{2}{*}{ Content } & \multicolumn{6}{|c|}{ Component } & \multirow{2}{*}{$\begin{array}{c}\text { Common } \\
\text { degree }\end{array}$} & \multirow{2}{*}{ CITC } & \multirow{2}{*}{$\begin{array}{l}\text { Cronbach' } \\
\alpha\end{array}$} \\
\hline & & 1 & 2 & 3 & 4 & 5 & 6 & & & \\
\hline B34 & Strategic alliance & 0.826 & 0.163 & & & 0.152 & 0.201 & 0.786 & 0.8390 & \\
\hline B32 & $\begin{array}{l}\text { Competitive } \\
\text { advantage }\end{array}$ & 0.812 & 0.159 & 0.104 & 0.187 & 0.150 & 0.142 & 0.776 & 0.8403 & \\
\hline B33 & $\begin{array}{r}\text { Access to new } \\
\text { technologies }\end{array}$ & 0.811 & 0.199 & & 0.123 & 0.127 & 0.143 & 0.759 & 0.8300 & \\
\hline B35 & $\begin{array}{l}\text { The effectiveness } \\
\text { of future } \\
\text { technical } \\
\text { cooperation }\end{array}$ & 0.785 & 0.156 & 0.113 & & & 0.242 & 0.735 & 0.8055 & \\
\hline B28 & $R \& D$ costs & 0.766 & 0.206 & & 0.120 & & & 0.666 & 0.7511 & 0.9479 \\
\hline B27 & $\mathrm{R} \& \mathrm{D}$ speed & 0.764 & 0.246 & 0.171 & & & & 0.701 & 0.7734 & \\
\hline B31 & $\begin{array}{l}\text { Ability of } \\
\text { independent } \\
\text { innovation }\end{array}$ & 0.762 & 0.203 & & 0.233 & 0.124 & 0.137 & 0.718 & 0.7815 & \\
\hline B29 & $\begin{array}{l}\text { New product } \\
\text { quality }\end{array}$ & 0.759 & 0.188 & 0.190 & & & 0.132 & 0.707 & 0.7780 & \\
\hline B30 & $\begin{array}{l}\text { NPD process } \\
\text { complexity }\end{array}$ & 0.735 & 0.212 & 0.176 & & 0.124 & & 0.661 & 0.7542 & \\
\hline B24 & $\begin{array}{l}\text { Technical risk of } \\
\text { R\&D }\end{array}$ & 0.227 & 0.875 & & 0.231 & 0.127 & & 0.923 & 0.5018 & \\
\hline B11 & $\begin{array}{l}\text { Increasingly } \\
\text { diversified } \\
\text { technology }\end{array}$ & 0.239 & 0.854 & 0.135 & 0.186 & & & 0.848 & 0.8668 & \\
\hline B13 & $\begin{array}{l}\text { Technological } \\
\text { innovation } \\
\text { degree }\end{array}$ & 0.226 & 0.853 & 0.128 & 0.180 & & & 0.834 & 0.8579 & \\
\hline B10 & $\begin{array}{l}\text { Technical } \\
\text { complexity }\end{array}$ & 0.266 & 0.839 & & & 0.148 & 0.138 & 0.827 & 0.8341 & \\
\hline B14 & $\begin{array}{l}\text { Technology } \\
\text { integration } \\
\text { degree }\end{array}$ & 0.280 & 0.829 & & & 0.111 & 0.141 & 0.808 & 0.8283 & 0.9172 \\
\hline B8 & $\begin{array}{l}\text { Technical } \\
\text { complexity } \\
\text { required by } \\
\text { product }\end{array}$ & 0.239 & 0.823 & 0.115 & 0.154 & & & 0.777 & 0.8190 & \\
\hline B7 & $\begin{array}{l}\text { Product } \\
\text { composition } \\
\text { complexity }\end{array}$ & & 0.800 & & & 0.175 & 0.147 & 0.712 & 0.7178 & \\
\hline B12 & $\begin{array}{c}\text { The degree of } \\
\text { Technology } \\
\text { uncertainty }\end{array}$ & 0.169 & 0.604 & & 0.323 & & & 0.523 & 0.5972 & \\
\hline
\end{tabular}




\begin{tabular}{|c|c|c|c|c|c|c|c|c|c|c|}
\hline \multirow{2}{*}{$\begin{array}{l}\text { Item } \\
\text { B6 }\end{array}$} & \multirow{2}{*}{$\begin{array}{l}\text { Content } \\
\begin{array}{c}\text { The number of } \\
\text { competitors }\end{array}\end{array}$} & \multicolumn{6}{|c|}{ Component } & \multirow{2}{*}{$\begin{array}{c}\begin{array}{c}\text { Common } \\
\text { degree }\end{array} \\
0.923\end{array}$} & \multirow{2}{*}{$\begin{array}{l}\text { CITC } \\
0.8941\end{array}$} & \multirow{2}{*}{$\begin{array}{l}\text { Cronbach's } \\
\alpha \\
\end{array}$} \\
\hline & & 0.144 & & 0.931 & & 0.160 & & & & \\
\hline B5 & $\begin{array}{r}\text { The scope for } \\
\text { competition }\end{array}$ & 0.178 & & 0.892 & & 0.168 & & 0.866 & 0.8465 & \\
\hline B4 & $\begin{array}{l}\text { Intensity of } \\
\text { competition }\end{array}$ & 0.117 & & 0.881 & & 0.143 & & 0.829 & 0.8111 & 0.8940 \\
\hline B3 & $\begin{array}{l}\text { Variety of } \\
\text { product } \\
\text { categories }\end{array}$ & 0.251 & 0.101 & 0.580 & 0.153 & 0.205 & & 0.622 & 0.5459 & \\
\hline B20 & $\begin{array}{l}\text { Difficulty to turn } \\
\text { to other } \\
\text { suppliers }\end{array}$ & 0.157 & 0.185 & & 0.838 & 0.205 & & 0.805 & 0.7627 & \\
\hline B23 & Supplier's power & 0.141 & 0.197 & & 0.825 & 0.203 & & 0.782 & 0.7294 & \\
\hline B19 & $\begin{array}{l}\text { Long-term } \\
\text { supplier } \\
\text { dependence }\end{array}$ & 0.182 & 0.160 & 0.144 & 0.778 & & & 0.775 & 0.7706 & 0.8886 \\
\hline B22 & $\begin{array}{l}\text { Technical } \\
\text { capacity of } \\
\text { suppliers }\end{array}$ & 0.173 & 0.156 & 0.129 & 0.770 & & & 0.764 & 0.7615 & \\
\hline B17 & $\begin{array}{l}\text { Enterprises' } \\
\text { belonging to } \\
\text { R\&D industry }\end{array}$ & 0.215 & 0.109 & 0.262 & 0.157 & 0.867 & 0.130 & 0.932 & 0.9415 & \\
\hline B15 & $\begin{array}{l}\text { Investing heavily } \\
\text { in } R \& D\end{array}$ & 0.220 & & 0.239 & 0.211 & 0.818 & & 0.846 & 0.8118 & 0.9134 \\
\hline B16 & $\begin{array}{l}\mathrm{R} \& \mathrm{D} \text { is the major } \\
\text { source of } \\
\text { competitive } \\
\text { advantage }\end{array}$ & 0.234 & 0.102 & 0.403 & & 0.703 & 0.142 & 0.756 & 0.7331 & \\
\hline B37 & $\begin{array}{l}\text { Government's } \\
\text { effort to } \\
\text { increase } \\
\text { investment }\end{array}$ & 0.242 & 0.114 & & & & 0.862 & 0.823 & 0.7718 & \\
\hline B36 & $\begin{array}{l}\text { National policy } \\
\text { emphasis on } \\
\text { innovation }\end{array}$ & 0.181 & & 0.132 & & & 0.831 & 0.754 & 0.6792 & 0.8398 \\
\hline B38 & $\begin{array}{l}\text { Policy incentives } \\
\text { on R\&D } \\
\text { investment }\end{array}$ & 0.248 & 0.111 & 0.107 & & 0.142 & 0.759 & 0.696 & 0.6633 & \\
\hline
\end{tabular}

\subsection{Analysis of Enabling Factors}

Based on the above rationale and literature review, we use SPSS11.5 and AMOS 7.0 to validate the enabling factors of SINPD and to ensure that the factor model 
established accordingly can fit in with the actual situation in Chinese enterprises.

Table 7 shows the Cronbach's $\alpha$ of each dimension are all above 0.70 , and the CITC of each item above 0.5 , showing that our scale has a good reliability, and the reliability of those corresponding latent variables are also acceptable.

Table 7 Cronbach's $\alpha$ of the Scales for SINPD Enabling Factors

\begin{tabular}{cccccccccc}
\hline $\begin{array}{c}\text { Operation } \\
\text { variable }\end{array}$ & CITC & $\begin{array}{c}\text { Cronbach's } \\
\alpha\end{array}$ & $\begin{array}{c}\text { Operation } \\
\text { variable }\end{array}$ & CITC & $\begin{array}{c}\text { Cronbach's } \\
\alpha\end{array}$ & $\begin{array}{c}\text { Operation } \\
\text { variable }\end{array}$ & CITC & $\begin{array}{c}\text { Cronbach's } \\
\alpha\end{array}$ \\
\hline $\mathrm{O}_{11}$ & 0.8978 & & $\mathrm{O}_{21}$ & 0.8407 & & $\mathrm{H}_{11}$ & 0.6464 & \\
$\mathrm{O}_{12}$ & 0.8756 & 0.9538 & $\mathrm{O}_{22}$ & 0.5299 & 0.8512 & $\mathrm{H}_{12}$ & 0.7822 & 0.8355 \\
$\mathrm{O}_{13}$ & 0.9375 & & $\mathrm{O}_{23}$ & 0.8203 & & $\mathrm{H}_{13}$ & 0.6700 & \\
$\mathrm{H}_{21}$ & 0.6441 & & $\mathrm{H}_{31}$ & 0.7816 & & $\mathrm{H}_{41}$ & 0.6792 & \\
$\mathrm{H}_{22}$ & 0.6572 & 0.7736 & $\mathrm{H}_{32}$ & 0.7643 & 0.8848 & $\mathrm{H}_{42}$ & 0.7718 & 0.8398 \\
$\mathrm{H}_{23}$ & 0.5309 & & $\mathrm{H}_{33}$ & 0.7841 & & $\mathrm{H}_{43}$ & 0.6633 & \\
$\mathrm{R}_{11}$ & 0.6883 & & $\mathrm{R}_{21}$ & 0.7390 & & $\mathrm{R}_{31}$ & 0.7893 & \\
$\mathrm{R}_{12}$ & 0.7312 & 0.8284 & $\mathrm{R}_{22}$ & 0.7527 & 0.8362 & $\mathrm{R}_{32}$ & 0.8126 & 0.8882 \\
$\mathrm{R}_{13}$ & 0.6414 & & $\mathrm{R}_{23}$ & 0.6073 & & $\mathrm{R}_{33}$ & 0.7473 & \\
$\mathrm{~A}_{11}$ & 0.7394 & & $\mathrm{~A}_{21}$ & 0.6785 & & $\mathrm{~A}_{31}$ & 0.6351 & \\
$\mathrm{~A}_{12}$ & 0.8518 & 0.8819 & $\mathrm{~A}_{22}$ & 0.6787 & 0.8273 & $\mathrm{~A}_{32}$ & 0.6351 & 0.7726 \\
$\mathrm{~A}_{13}$ & 0.7272 & & $\mathrm{~A}_{23}$ & 0.6994 & & - & - & \\
\hline
\end{tabular}

Amos 7.0 software was used to conduct a CFA on enabling factors of SINPD collected by the 149 questionnaires. Table 8 presents the parameters estimation results of all four measuring models. All factors loading are greater than 0.5, indicating that the scale has good construct validity.

The commonly used fit indexes for model assessment in structural equation model analysis include GFI, AGFI, NFI or TLI, CFI and IFI (Van Echtelt and Wynstra, 2001). When the values of all these indexes are bigger than 0.9, it indicates that a model has acceptable fitness. A RMSEA smaller than 0.05 means that a model has very good fitness, while between 0.05 and 0.08 means good fitness, and between 0.08 and 0.10 means acceptable fitness, bigger than 0.10 poor fitness. As a rule, the value of $\chi^{2} / \mathrm{df}$ shall be smaller than 5 , or even than 3 . When considering the fitness of model, also should relate it with the values of critical ratio (CR). $P$ value is also considered when accessing the fitness of a model.

$\mathrm{CR}$ and $P$ values in Table 9 indicate that all the variables of enabling factors in SINPD questionnaire are significant at 0.01 levels, showing that the model has a good convergent validity. The value of $\chi^{2} / \mathrm{df}$ are all less than 5 (the value of organization management is less than 3). Except the AGFI of human resource 
Table 8 Validity Analysis of Scales for SINPD Enabling Factors

\begin{tabular}{|c|c|c|c|c|c|c|c|}
\hline \multirow[t]{2}{*}{ Latent variable } & \multirow[t]{2}{*}{$\begin{array}{r}\text { Observed } \\
\text { variable }\end{array}$} & \multicolumn{2}{|c|}{ Whole sample } & \multirow[t]{2}{*}{ Latent variable } & \multirow[t]{2}{*}{$\begin{array}{r}\text { Observed } \\
\text { variable }\end{array}$} & \multicolumn{2}{|c|}{ Whole sample } \\
\hline & & $\begin{array}{l}\text { Factor } \\
\text { loading } \\
(\lambda)\end{array}$ & $\begin{array}{l}\text { Residual } \\
(\delta)\end{array}$ & & & $\begin{array}{l}\text { Factor } \\
\text { loading } \\
(\lambda)\end{array}$ & $\begin{array}{c}\text { Residual } \\
(\delta)\end{array}$ \\
\hline \multirow{3}{*}{$\begin{array}{l}\mathrm{O}_{1} \text { : Organizational } \\
\text { structure of } \mathrm{R} \& \mathrm{D} \\
\text { departments }\end{array}$} & $\mathrm{O}_{11}$ & 0.93 & 0.86 & \multirow{3}{*}{$\begin{array}{l}\mathrm{R}_{1} \text { : Mutual } \\
\text { trust }\end{array}$} & $\mathrm{R}_{11}$ & 0.80 & 0.65 \\
\hline & $\mathrm{O}_{12}$ & 0.90 & 0.81 & & $\mathrm{R}_{12}$ & 0.85 & 0.72 \\
\hline & $\mathrm{O}_{13}$ & 0.98 & 0.97 & & $\mathrm{R}_{13}$ & 0.71 & 0.60 \\
\hline \multirow{3}{*}{$\begin{array}{l}\mathrm{O}_{2}: \text { New product } \\
\text { development } \\
\text { team }\end{array}$} & $\mathrm{O}_{21}$ & 0.98 & 0.97 & \multirow{3}{*}{$\begin{array}{l}\mathrm{R}_{2} \text { : } \\
\text { Information } \\
\text { exchange } \\
\text { systems }\end{array}$} & $\mathrm{R}_{21}$ & 0.85 & 0.73 \\
\hline & $\mathrm{O}_{22}$ & 0.54 & 0.29 & & $\mathrm{R}_{22}$ & 0.84 & 0.71 \\
\hline & $\mathrm{O}_{23}$ & 0.93 & 0.87 & & $\mathrm{R}_{23}$ & 0.71 & 0.61 \\
\hline \multirow{3}{*}{$\begin{array}{l}\mathrm{H}_{1}: \text { Education and } \\
\text { training level }\end{array}$} & $\mathrm{H}_{11}$ & 0.77 & 0.60 & \multirow{3}{*}{$\begin{array}{l}\mathrm{R}_{3}: \text { Cultural } \\
\text { compatibility }\end{array}$} & $\mathrm{R}_{31}$ & 0.85 & 0.72 \\
\hline & $\mathrm{H}_{12}$ & 0.84 & 0.71 & & $\mathrm{R}_{32}$ & 0.89 & 0.79 \\
\hline & $\mathrm{H}_{13}$ & 0.79 & 0.62 & & $\mathrm{R}_{33}$ & 0.83 & 0.69 \\
\hline \multirow{3}{*}{$\begin{array}{l}\mathrm{H}_{2} \text { : Previous } \\
\text { experience of } \\
\text { cooperation }\end{array}$} & $\mathrm{H}_{21}$ & 0.78 & 0.61 & \multirow{3}{*}{$\begin{array}{l}\mathrm{A}_{1}: \text { Suppliers } \\
\text { technical } \\
\text { capabilities }\end{array}$} & $\mathrm{A}_{11}$ & 0.81 & 0.66 \\
\hline & $\mathrm{H}_{22}$ & 0.77 & 0.59 & & $\mathrm{~A}_{12}$ & 0.94 & 0.88 \\
\hline & $\mathrm{H}_{23}$ & 0.66 & 0.44 & & $\mathrm{~A}_{13}$ & 0.80 & 0.64 \\
\hline \multirow{3}{*}{$\begin{array}{l}\mathrm{H}_{3}: \text { Perceived trust } \\
\text { capabilities }\end{array}$} & $\mathrm{H}_{31}$ & 0.86 & 0.74 & \multirow{3}{*}{$\begin{array}{l}\mathrm{A}_{2}: \text { The } \\
\text { manufacturer } \\
\text { commitment }\end{array}$} & $\mathrm{A}_{21}$ & 0.75 & 0.56 \\
\hline & $\mathrm{H}_{32}$ & 0.82 & 0.68 & & $\mathrm{~A}_{22}$ & 0.72 & 0.52 \\
\hline & $\mathrm{H}_{33}$ & 0.86 & 0.74 & & $\mathrm{~A}_{23}$ & 0.87 & 0.75 \\
\hline \multirow{3}{*}{$\begin{array}{l}\mathrm{H}_{4}: \text { Technical } \\
\text { expertise }\end{array}$} & $\mathrm{H}_{41}$ & 0.73 & 0.54 & \multirow{3}{*}{$\begin{array}{l}\mathrm{A}_{3}: \\
\text { Geographic } \\
\text { location }\end{array}$} & $\mathrm{A}_{31}$ & 0.82 & 0.68 \\
\hline & $\mathrm{H}_{42}$ & 0.83 & 0.69 & & $\mathrm{~A}_{32}$ & 0.77 & 0.59 \\
\hline & $\mathrm{H}_{43}$ & 0.85 & 0.69 & & - & - & - \\
\hline
\end{tabular}

quality $(\mathrm{H})$, cooperate relationship (R) and interact attraction (A) is $0.891,0.895$ and 0.896, respectively, most indexes of GFI、AGFI、TLI、CFI、IFI are bigger than 0.9; The value of RMSEA is small than 0.1. All these indexes indicate that the model has a good data fitness.

Table 9 Confirmatory Factor Analysis Result of Enabling Factors

\begin{tabular}{|c|c|c|c|c|c|}
\hline Dimension & Latent variable & $\begin{array}{r}\text { Observed } \\
\text { variable }\end{array}$ & $\mathrm{CR}$ & $P$ & Fit index \\
\hline \multirow{6}{*}{$\begin{array}{r}\text { Organizational } \\
\text { management }\end{array}$} & \multirow{3}{*}{$\begin{array}{l}\text { Organizational } \\
\text { structure of R\&D } \\
\text { department }\end{array}$} & $\mathrm{O}_{11}$ & - & - & \multirow{6}{*}{$\begin{array}{l}\chi^{2}=14.3 ; \chi^{2} / \mathrm{df}=1.788 \\
\text { GFI }=0.989 ; \text { AGFI } \\
0.972 ; \text { TLI }=0.995 ; \\
\text { CFI }=0.997 ; \text { IFI }= \\
0.997 ; \text { RMSEA }= \\
0.043\end{array}$} \\
\hline & & $\mathrm{O}_{12}$ & 31.830 & $* * *$ & \\
\hline & & $\mathrm{O}_{13}$ & 41.349 & $* * *$ & \\
\hline & \multirow{3}{*}{$\begin{array}{l}\text { New product } \\
\text { development } \\
\text { team }\end{array}$} & $\mathrm{O}_{21}$ & - & - & \\
\hline & & $\mathrm{O}_{22}$ & 12.313 & $* * *$ & \\
\hline & & $\mathrm{O}_{23}$ & 26.133 & $* * *$ & \\
\hline
\end{tabular}


(Continued)

\begin{tabular}{|c|c|c|c|c|c|}
\hline Dimension & Latent variable & $\begin{array}{r}\text { Observed } \\
\text { variable }\end{array}$ & $\mathrm{CR}$ & $P$ & Fit index \\
\hline \multirow{12}{*}{$\begin{array}{l}\text { Human } \\
\text { resources } \\
\text { quality }\end{array}$} & \multirow{3}{*}{$\begin{array}{l}\text { Education and } \\
\text { training level }\end{array}$} & $\mathrm{H}_{11}$ & - & - & \multirow{12}{*}{$\begin{array}{l}\chi^{2}=183.8 ; \chi^{2} / \mathrm{df}= \\
3.829 ; \mathrm{GFI}=0.933 ; \\
\mathrm{AGFI}=0.901 ; \mathrm{TLI}= \\
0.945 ; \mathrm{CFI}=0.960 ; \\
\text { IFI }=0.961 ; \\
\text { RMSEA }=0.081\end{array}$} \\
\hline & & $\mathrm{H}_{12}$ & 17.931 & $* * *$ & \\
\hline & & $\mathrm{H}_{13}$ & 16.672 & $* * *$ & \\
\hline & \multirow{3}{*}{$\begin{array}{l}\text { Previous } \\
\text { experience of } \\
\text { cooperation }\end{array}$} & $\mathrm{H}_{21}$ & - & - & \\
\hline & & $\mathrm{H}_{22}$ & 17.167 & $* * *$ & \\
\hline & & $\mathrm{H}_{23}$ & 14.393 & $* * *$ & \\
\hline & \multirow{3}{*}{$\begin{array}{l}\text { Perceived trust } \\
\text { capabilities }\end{array}$} & $\mathrm{H}_{31}$ & - & - & \\
\hline & & $\mathrm{H}_{32}$ & 21.460 & $* * *$ & \\
\hline & & $\mathrm{H}_{33}$ & 23.063 & $* * *$ & \\
\hline & \multirow{3}{*}{ Technical expertise } & $\mathrm{H}_{41}$ & - & - & \\
\hline & & $\mathrm{H}_{42}$ & 16.412 & $* * *$ & \\
\hline & & $\mathrm{H}_{43}$ & 16.416 & $* * *$ & \\
\hline \multirow{9}{*}{$\begin{array}{l}\text { Cooperation } \\
\text { relationship }\end{array}$} & \multirow{3}{*}{$\begin{array}{l}\text { Mutual trust } \\
\text { information }\end{array}$} & $\mathrm{R}_{11}$ & - & - & \multirow{9}{*}{$\begin{array}{l}\chi^{2}=110.56 ; \chi^{2} / \mathrm{df}= \\
4.604 ; \mathrm{GFI}=0.944 ; \\
\text { AGFI }=0.905 ; \\
\text { TLI }=0.947 ; \\
\text { CFI }=0.965 ; \\
\text { IFI }=0.965 ; \\
\text { RMSEA }=0.092\end{array}$} \\
\hline & & $\mathrm{R}_{12}$ & 17.430 & $* * *$ & \\
\hline & & $\mathrm{R}_{13}$ & 14.764 & $* * *$ & \\
\hline & \multirow{6}{*}{$\begin{array}{l}\text { Cultural } \\
\text { compatibility }\end{array}$} & $\mathrm{R}_{21}$ & - & - & \\
\hline & & $\mathrm{R}_{22}$ & 20.263 & $* * *$ & \\
\hline & & $\mathrm{R}_{23}$ & 16.232 & $* * *$ & \\
\hline & & $\mathrm{R}_{31}$ & - & - & \\
\hline & & $\mathrm{R}_{32}$ & 23.154 & $* * *$ & \\
\hline & & $\mathrm{R}_{33}$ & 20.863 & $* * *$ & \\
\hline \multirow{8}{*}{$\begin{array}{l}\text { Mutual } \\
\text { attraction }\end{array}$} & \multirow{3}{*}{$\begin{array}{l}\text { Suppliers technical } \\
\text { capabilities }\end{array}$} & $\mathrm{A}_{11}$ & - & - & \multirow{8}{*}{$\begin{array}{l}\chi^{2}=90.4 ; \chi^{2} / \mathrm{df}=4.183 ; \\
\mathrm{GFI}=0.951 ; \mathrm{AGFI}= \\
0.906 ; \mathrm{TLI}=0.944 ; \\
\mathrm{CFI}=0.966 ; \mathrm{IFI}= \\
0.966 ; \text { RMSEA }= \\
0.098\end{array}$} \\
\hline & & $\mathrm{A}_{12}$ & 22.192 & $* * *$ & \\
\hline & & $\mathrm{A}_{13}$ & 18.931 & $* * *$ & \\
\hline & \multirow{3}{*}{$\begin{array}{l}\text { The manufacturer } \\
\text { commitment }\end{array}$} & $\mathrm{A}_{21}$ & - & - & \\
\hline & & $\mathrm{A}_{22}$ & 14.899 & $* * *$ & \\
\hline & & $\mathrm{A}_{23}$ & 18.015 & $* * *$ & \\
\hline & \multirow{2}{*}{$\begin{array}{l}\text { Geographic } \\
\text { location }\end{array}$} & $\mathrm{A}_{31}$ & - & - & \\
\hline & & $\mathrm{A}_{32}$ & 17.562 & $* * *$ & \\
\hline
\end{tabular}

Note: *** denotes $P<0.01$; “-“" means no value, indicating situations in which standardized regression coefficients are set as 1 .

Based on the above analysis, 4 dimensions of enabling factors are proposed: organization management, human resource quality, cooperation relationship and interact attraction, including 12 factors (i.e., organizational structure of the 
relevant departments of $\mathrm{R} \& \mathrm{D}$, new product development team, education and training level, previous experience of cooperation, perceived trust capabilities, technical expertise, mutual trust, information exchange systems, cultural compatibility, suppliers technical capabilities, the manufacturer commitment and geographic location).

\section{Theoretical Framework}

In reality, the implementation of SINPD varies in different enterprises. Below, we are going to introduce enterprise type as a moderator to further explore the function mechanism of the influencing factors on SINPD. Fig. 4 is a comprehensive theoretical model for influencing factors of SINPD.

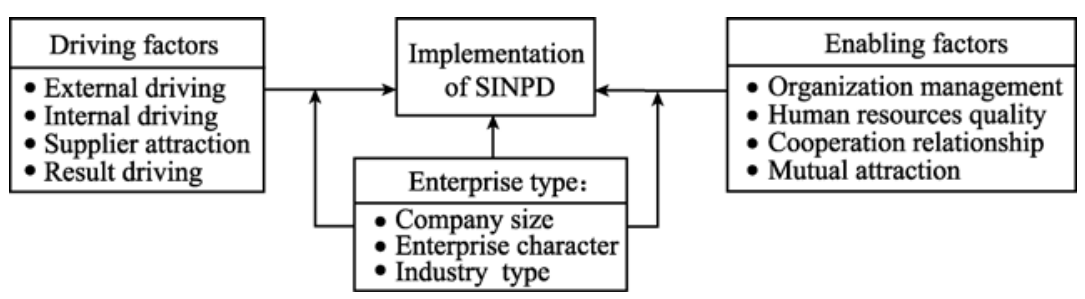

Fig. 4 Model for Influencing Factors of SINPD

This paper divides enterprises by their size (i.e., large, medium and small enterprises), nature (i.e., state-owned, private, non-state-owned joint stock, joint ventures, foreign-owned, and other types), and industry. To better compare different enterprises, we grouped state-controlled, private and non-statecontrolled joint-stock as domestic-funded enterprises, grouped joint ventures, foreign-owned enterprises, and other forms of enterprise as foreign-funded enterprises. Sampled enterprises can be classified into four types of industry, namely ordinary machinery, special equipment manufacturing, transportation equipment manufacturing, electrical machinery and equipment manufacturing. Ordinary machinery manufacturing includes metal processing, universal equipment, boilers and original motivation. The technical standardization is high in these industries, but the speed of $R \& D$ is slow. Special equipment Manufacturing includes metallurgy, mine, petrochemical and rubber manufacturing, which are mainly made according to customers' demand, also require a low technological content. Transportation equipment manufacturing includes automobile manufacturing and aerospace manufacturing. The product updating speed in these industries are rapid due to increasingly fierce market competition, leading to a high requirement of R\&D technology in these industries. Likewise, manufacture of electrical machinery and equipment, 
including household electrical appliances, electronic products, etc., also requires strong high R\&D capabilities. Based on their requirements on R\&D capabilities, we divided the manufacturing industry into technology-intensive manufacturing and general manufacturing enterprises.

\subsection{Enterprise Type and the Implementation of SINPD}

Implementation of SINPD refers to supplier's involvement time and degree. Involvement time includes early involvement, metaphase involvement and anaphase involvement. Early involvement mainly refers to the supplier involvement in phase of product conceiving and planning stage, technical evaluation stage, product concept stage and product design stage. The metaphase involvement refers to supplier involvement in the product process phase. Anaphase involvement is defined as supplier involvement in prototype establishment and limited trial-manufacture stage. Involvement degree can be divided into low, medium, and high level. Low degree involvement refers to manufacturers only considering supplier's advice in new product development. Medium degree involvement refers to making supplier part of the new product development activities. High degree involvement refers to the fact that manufacturers allow suppliers to complete parts manufacturing or sub-system design independently in accordance with the requirements of their buyers (manufacturing enterprises).

In implementing SINPD, manufacturers tend to invite suppliers to participate in new product development in accordance with their practical needs, making it difficult to investigate SINPD. However, there are still some commonly-obeyed rules in the implementation of SINPD.

(1) Implementation of SINPD in different sized enterprises. Compared with the small and medium enterprises, large enterprise have stronger technological capabilities, more abundant R\&D capital and thus more inclined to a early and high degree involvement. On the contrary, small and medium enterprises, in order to avoid risks, are more likely to choose later and lower degree involvement. We therefore propose Hypothesis 1 as follows:

H1 Compared with small and medium enterprises, large enterprises are more likely to choose early and high degree SINPD.

(2) Implementation of SINPD in domestic/foreign-funded enterprises. SINPD mode originated in Japan and later flourished and matured in Europe and USA. Since its later introduction into China, domestic enterprises are very likely to be lagged far behind foreign-funded enterprises due to their weaker sense of cooperation and the lack of mutual trust mechanism. We thus assume that:

H2 On the whole, domestic-funded enterprises are later in involvement time 
and lower in involvement degree than their foreign-funded counterparts.

(3) The implementation of SINPD in enterprises from different industries. Clearly, technology-intensive manufacturing enterprises, in order to respond rapidly to market changes, have greater need to make better use of supplier technology than their counterparts in non-technology-intensive industries. Therefore, they will have earlier supplier involvement and higher involvement degree. Thus

H3 Comparing with enterprises in the general manufacturing industry group, enterprises in the technology-intensive manufacturing industries are more likely to have earlier supplier involvement and higher involvement degree.

\subsection{Enterprise Type and Driving Factors of SINPD}

Six key driving factors of SINPD have extracted in the preliminary study, namely external competition pressure, national innovation policy guidance, product complexity, technology integration, $R \& D$ dependence, supplier dependence, expected obtain performance. Implementation of SINPD is the result of a variety of driving factors. In order to effectively identify the motivations behind different enterprises' implementation of SINPD, we need to further examine the influence of different driving factors on SINPD implementation.

(1) Impact of driving factors on SINPD implementation in different-sized enterprises. By comparison, large enterprises tend to take the initiative to implement SINPD to guarantee long-term competitive advantages. The national innovation policy guidance, product complexity, technology integration, R\&D dependence have larger effect degree on large enterprises than on small and medium-sized enterprises. Large enterprises also focus more on long-term performance. On the contrary, small and medium enterprises, due to their smaller size, are more vulnerable to external competition pressures, resulting in more reliance on suppliers and more emphasis on short-term performance. Based on this rationale, we put forward the hypothesis 4 :

H4 The implementation of SINPD in large enterprises are more likely to be driven by national innovation policy guidance, product complexity, technology integration, R\&D dependence and expectation of long-term performance; while the implementation of SINPD in small and medium enterprises are more likely to be driven by external competition pressures, supplier dependence and expectation of short-term performance.

(2) Impact of driving factors on SINPD implementation in different types of enterprises. Compared with foreign-funded enterprises, the R\&D capability of 
domestic-funded enterprises is relatively weak. Most of domestic-funded enterprises are in the manufacturing business. Their implementation of SINPD is, in most cases, a direct result of national innovation policy guidance. Accordingly, domestic-funded enterprises pay more attention to short-term performance. In contrast to their Chinese counterparts, most of the foreign-funded enterprises are more mature in terms of R\&D capabilities. The implementation of SINPD in these enterprises is usually a result of external competition. As a lot of foreign-funded enterprises are $\mathrm{R} \& \mathrm{D}$-driven enterprises, the driving factors of their implementation of SINPD are more likely to be product complexity, technology integration and R\&D dependence. Thus, we assume that,

H5 The implementation of SINPD in domestic-funded enterprises are more likely to be driven by national innovation policy and performance expectation, while the implementation of SINPD in foreign-funded enterprises are more likely to be driven by external competition pressures, product complexity, technology integration and $\mathrm{R} \& \mathrm{D}$ dependence.

(3) Impact of driving factors on the SINPD implementation in different industries. Obviously, enterprises in technology-intensive industries need to continuously develop new technologies and products to win market share, resulting in enormous competition pressure. Due to resource limitation, these enterprises tend to focus on cultivation of core competitiveness and outsource other non-core parts to their suppliers. Therefore, the implementation of SINPD in these enterprises is mainly driven by external competition pressure, product complexity, technology integration, supplier dependence. By comparison, the implementations of SINPD in enterprises in general manufacturing industries are more likely to be driven by national innovation policy guidance and performance expectation. We therefore develop hypothesis 6 as below:

H6 The implementation of SINPD in enterprises of technology-intensive industries are more likely to be driven by external competition pressures, product complexity, technology integration, supplier dependence; while the implementation of SINPD in enterprises of general manufacturing industries are more likely to be driven by national innovation policy guidance and performance expectation.

\subsection{Enterprise Type and Enabling Factors of SINPD}

As above, enabling factors vary with different types of enterprises. To gain a better understanding of how to implement SINPD and further improve SINPD effectiveness, we need to further study the specific impact of different enabling factors on SINPD implementation. Extant literature has proposed four dimensions of enabling factors for SINPD, namely organization management, 
human resource quality, cooperation relationship and interact attraction.

Organization management refers to the impact of organizational structure of $R \& D$ departments and the organization and management methods of the new product development teams on the communication and cooperation capabilities of $\mathrm{R} \& \mathrm{D}$ staffs. If a new product development team is project-oriented, it is more beneficial to the cooperation among team members; on the contrary, if the team is function-oriented, it will hinder rather than promote cooperation among team members. Thus, effective organizational management is a basis of the SINPD management.

The quality of human resource quality is an important factor which can influence the effectiveness of SINPD implementation. The experience of cooperation, appropriate training or education, the degree of technical expertise, and the ability to win trust from others are the key factors for effective SINPD partnership. Mutual trust, information exchange system and culture compatibility are the main components of this partnership. Among them, mutual trust is vital to successful cooperation. In other words, if enterprise atmosphere is open and supportive of mutual trust, it will strengthen the cooperation among all partners involved in SINPD.

New product development is the information collection of a series of activities, which are different in contents, frequency and manner. All supplier involvement activities consists of information elements, which means that the database access or other means of information exchange methods are needed to carry out technology or supplier market profile. From the aspect of culture compatibility, three factors have been found influencing the cooperation between manufacturers and suppliers, namely value sharing, operation model and conflict resolution methods. Failure of match of these factors between enterprises and suppliers will lead to unsuccessful involvement of suppliers. Interact attraction is an interactive process of cooperation between manufacturer and supplier, cooperation attraction of supplier mainly refers to the technical capacity of suppliers; and cooperation attraction of manufacturers mainly refers to manufacturer commitment, such as the tendency of long-term cooperation. Manufacturers in close relationships with suppliers geographically can enhance the mutual attraction between the two parties.

(1) Impact of enabling factors on the SINPD implementation in different-sized enterprises. Compared with large enterprises, small and medium enterprises tend to have higher level of complexity, adopt team-work approach, and be more project-oriented: all these are beneficial to more closely-knit cooperation among R\&D team members. Large enterprises have more managerial levels, making it more difficult for people at different managerial levels to cooperate, which in turn discourage suppliers from participating in new product development. However, large enterprises have advantages over small and medium enterprises 
in the quality of human resources, more developed information exchange mechanism and culture management system. To summarize, small and medium enterprises are more likely to form sound cooperation relationship based on inter-dependence and mutual trust, while big enterprises are more likely to have greater attraction to suppliers due to their stronger technology capabilities. Thus:

H7 In terms of the enabling factors of SINPD, when implementing SINPD, large enterprises are likely to be affected by the quality of human resources, cooperation relationship and mutual attraction, whereas small and medium enterprises are more likely to be affected by organizational management.

(2) Impact of enabling factors on SINPD implementation in different types of enterprises. Compared with domestic-funded enterprises, foreign-funded enterprises tend to have higher technological capabilities and greater attraction. There are no significant differences between the two types of enterprises in other aspects. Therefore, we put forward the hypothesis 8:

H8 In terms of the enabling factors of SINPD, when implementing SINPD, foreign-funded enterprises are more likely to be affected by mutual attraction. Except the factor of mutual attraction, there is no significant difference between foreign-funded enterprises and domestic-funded enterprises.

(3) Impact of enabling factors on SINPD implementation of enterprises in different industries. As a rule, enterprises in technology-intensive industries are more likely to form R\&D project teams, which make it more suitable for SINPD in terms of organizational management. In addition, enterprises in technology-intensive industries are more experienced in cooperation with outsiders, and have higher quality human resources and mutual relationship with their partners. Therefore, we assume that they would have greater appealing to suppliers:

H9 In terms of the enabling factors of SINPD, when implementing SINPD, enterprises in technology-intensive industries are more likely to be affected by organizational management, human resources quality, cooperation relationship and mutual attraction.

\section{Results and Discussion}

\subsection{The Sample}

In order to ensure the representativeness of our participants, our final questionnaires were sent to more than 100 enterprises in 11 different provinces in China (i.e., Shaanxi, Gansu, Shanxi, Ningxia, Henan, Shandong, Zhejiang, Jiangsu, Liaoning, Jilin, Sichuan). As noted earlier, 429 valid questionnaires 
were returned out of a total of 550 questionnaires delivered.

The natures of ownership of sampled enterprises include: state-controlled enterprises $(45.7 \%)$, private enterprises (32.4\%), joint-stock (non-state-controlled) enterprises $(13.1 \%)$, joint ventures enterprises $(7.0 \%)$, foreign-owned enterprises $(0.1 \%)$, and other forms of enterprise (1.6\%). The type of industries include: automobile manufacturing (15.2\%), aviation and aerospace manufacturing $(1.6 \%)$, machinery manufacturing $(13.1 \%)$, electronic and communications equipment manufacturing (11.4\%), electrical equipment manufacturing $(23.5 \%)$, medical equipment and instruments manufacturing (3.5\%), metallurgy and construction equipment manufacturing $(19.3 \%)$, software industry $(0.7 \%)$, other manufacturing (11.7\%). The participants were mainly from R\&D department or other departments directly related R\&D, such as R\&D department (28.2\%), technology management department $(28.0 \%)$, design \& craft department $(7.0 \%)$, purchase department (14.7\%), development plan department (11.4\%), manufacturing department $(10.0 \%)$ or other department $(0.7 \%)$. Therefore, we hold that the questionnaire can reflect the actual situation of SINPD among Chinese enterprises. In addition, based on the number of employees, sales revenue and total assets, the firm size can be divided into large, medium and small enterprises. The composition and R\&D investment of these three different-sized enterprises are shown in Table 10.

Table 10 Composition of Different Sized Enterprises and Their R\&D Input

\begin{tabular}{|c|c|c|c|c|c|c|c|c|}
\hline \multirow[b]{2}{*}{$\begin{array}{l}\text { Enterprise } \\
\text { size }\end{array}$} & \multicolumn{3}{|c|}{ Division criterion } & \multirow[b]{2}{*}{$\begin{array}{l}\text { Number of } \\
\text { enterprises }\end{array}$} & \multirow[b]{2}{*}{ Percentage } & \multicolumn{3}{|c|}{ R\&D Investment (\%) } \\
\hline & $\begin{array}{l}\text { No. of } \\
\text { employees }\end{array}$ & $\begin{array}{l}\text { Sales } \\
\text { revenue } \\
\text { (Yuan) }\end{array}$ & $\begin{array}{l}\text { Total } \\
\text { assets } \\
\text { (Yuan) }\end{array}$ & & & Min & Max & Average \\
\hline $\begin{array}{l}\text { Large } \\
\text { enterprise }\end{array}$ & over 2000 & $\begin{array}{r}\text { over } 300 \\
\text { million }\end{array}$ & $\begin{array}{r}\text { over } 400 \\
\text { million }\end{array}$ & 175 & $40.8 \%$ & 0.1 & 37 & 3.51 \\
\hline $\begin{array}{l}\text { Medium } \\
\text { enterprise }\end{array}$ & $300-2000$ & $\begin{array}{l}30-300 \\
\text { million }\end{array}$ & $\begin{array}{l}40-400 \\
\text { million }\end{array}$ & 138 & $32.2 \%$ & 0.1 & 30 & 8.95 \\
\hline $\begin{array}{l}\text { Small } \\
\text { enterprise }\end{array}$ & under 300 & $\begin{array}{r}\text { under } 30 \\
\text { million }\end{array}$ & $\begin{array}{r}\text { under } 30 \\
\text { million }\end{array}$ & 116 & $27.0 \%$ & 0.1 & 40 & 10.19 \\
\hline Total & - & - & - & 429 & $100.0 \%$ & 0.1 & 40 & 7.06 \\
\hline
\end{tabular}

Table 10 shows that the ratio of R\&D investment to sales revenue of all enterprises is $7.06 \%$ on average, showing that sampled enterprises have a rather high level of R\&D investment. Particularly, sampled small and medium-sized enterprises all have greater $R \& D$ investment than that of large enterprises.

Table 11 presents the situation of SINPD in sampled enterprises. As shown, all participating enterprises have implemented SINPD. 
Table 11 Descriptive Statistics of SINPD Implementation in Sampled Enterprises

\begin{tabular}{|c|c|c|c|}
\hline $\begin{array}{l}\text { SINPD } \\
\text { implementation }\end{array}$ & Items & Mean & $\begin{array}{c}\text { Standard } \\
\text { deviation }\end{array}$ \\
\hline \multirow{6}{*}{$\begin{array}{l}\text { Involvement time } \\
\text { (IVT) }\end{array}$} & $\begin{array}{l}\text { IVT1: Supplier participates in new product } \\
\text { development at the idea development and } \\
\text { planning stage }\end{array}$ & 4.3124 & 0.0769 \\
\hline & $\begin{array}{l}\text { IVT2: Supplier participates in new product } \\
\text { development at the product technical assessment } \\
\text { stage }\end{array}$ & 4.3753 & 0.0742 \\
\hline & $\begin{array}{l}\text { IVT3: Supplier participates in new product } \\
\text { development at the product conception stage }\end{array}$ & 4.2657 & 0.0776 \\
\hline & $\begin{array}{l}\text { IVT4: Supplier participates in new product } \\
\text { development at the product design stage }\end{array}$ & 4.4918 & 0.0747 \\
\hline & $\begin{array}{l}\text { IVT5: Supplier participates in new product } \\
\text { development at the product process stage }\end{array}$ & 4.6154 & 0.0718 \\
\hline & $\begin{array}{l}\text { IVT6: Supplier participates in new product } \\
\text { development at prototype establishment and trial } \\
\text { manufacturing stage }\end{array}$ & 4.7716 & 0.0720 \\
\hline \multirow{3}{*}{$\begin{array}{l}\text { Involvement } \\
\text { degree (IVD) }\end{array}$} & $\begin{array}{l}\text { IVD1: When developing new products, enterprises } \\
\text { only take advice from suppliers into } \\
\text { consideration }\end{array}$ & 4.4359 & 0.0829 \\
\hline & $\begin{array}{l}\text { IVD2: Suppliers are officially involved in } \\
\text { manufacturer's development of new products }\end{array}$ & 4.4103 & 0.0764 \\
\hline & $\begin{array}{l}\text { IVD3: Suppliers complete manufacturing parts or } \\
\text { sub-system design independently in accordance } \\
\text { with the requirements of their buyers }\end{array}$ & 4.7413 & 0.0726 \\
\hline
\end{tabular}

As shown in Table 11, sampled enterprises are of wide representation of Chinese enterprises in terms of enterprise type, participants, status quo of R\&D and SINPD.

\subsection{Reliability and Validity}

As shown in Table 12, the CITC values of all variables are all greater than 0.5 , Cronbach's $\alpha$ values of all subscales are above 0.70 , which means the reliability of the scale is acceptable. And the reliability of using these observation variables to measure the corresponding latent variable is acceptable.

Amos 7.0 was used for structuring validity analysis of the scale in order to test whether the factor structure is appropriate. The fitness indices of all driving and enabling factors are shown in Table 13.

Table 13 shows that all indices are within a reasonable range, so the model fits well with the data, indicating a satisfactory structural validity of our questionnaire. 
Table 12 Cronbach's $\alpha$ of Questionnaire

\begin{tabular}{|c|c|c|c|c|}
\hline Item & Factor & Number & CITC & Cronbach's $\alpha$ \\
\hline \multirow{2}{*}{$\begin{array}{l}\text { SINPD } \\
\text { implementation }\end{array}$} & Involvement time & 6 & 0.9144 & \multirow{2}{*}{0.9146} \\
\hline & Involvement degree & 3 & 0.7704 & \\
\hline \multirow{6}{*}{ Driving factors } & External competition pressure & 4 & 0.8940 & \multirow{6}{*}{0.9428} \\
\hline & National innovation policy guidance & 3 & 0.8398 & \\
\hline & $\begin{array}{l}\text { Product complexity, technology } \\
\text { integration }\end{array}$ & 8 & 0.9172 & \\
\hline & R\&D dependence & 3 & 0.9134 & \\
\hline & Supplier dependence & 4 & 0.8886 & \\
\hline & Expected obtain performance & 9 & 0.9479 & \\
\hline \multirow{5}{*}{ Enabling factors } & Organization management & 6 & 0.7495 & \multirow{5}{*}{0.9545} \\
\hline & Human resource quality & 12 & 0.9347 & \\
\hline & Cooperate relationship & 9 & 0.9149 & \\
\hline & Mutual attraction & 8 & 0.9099 & \\
\hline & Management actions & 18 & 0.9249 & \\
\hline
\end{tabular}

Table 13 Goodness-of-Fit of Confirmatory Factor Analysis of the Influencing Factors

\begin{tabular}{lccccccccc}
\hline Goodness-of-fit & $\chi^{2}$ & $\mathrm{df}$ & $\chi^{2} / \mathrm{df}$ & RMSEA & GFI & AGFI & CFI & IFI & TLI \\
\hline Driving factors & 1647.313 & 428 & 3.849 & 0.079 & 0.989 & 0.940 & 0.938 & 0.939 & 0.920 \\
Enabling factors & 1484.888 & 545 & 2.725 & 0.063 & 0.938 & 0.912 & 0.920 & 0.918 & 0.913 \\
\hline
\end{tabular}

6.3 Relationship between Enterprises Characteristics and Implementation of SINPD

To test H1, H2, H3, SPSS 11.5 was used to describe statistically SINPD participation (i.e., involvement time and degree). Table 14, 15, 16 shows the SINPD involvement of different sampled enterprises, respectively.

Table 14 Descriptive Statistics of SINPD Implementation in Different Sized Enterprises

\begin{tabular}{|c|c|c|c|c|c|c|c|c|c|c|}
\hline \multirow{2}{*}{$\begin{array}{l}\text { Company } \\
\text { size }\end{array}$} & \multirow{2}{*}{ Obs. } & \multicolumn{6}{|c|}{ Involvement time } & \multicolumn{3}{|c|}{ Involvement degree } \\
\hline & & IVT1 & IVT2 & IVT3 & IVT4 & IVT5 & IVT6 & IVD1 & IVD2 & IVD3 \\
\hline $\begin{array}{l}\text { Large } \\
\text { enterprises }\end{array}$ & 175 & 4.46 & 4.46 & 4.21 & 4.57 & 4.66 & 4.84 & 4.33 & 4.35 & 4.85 \\
\hline $\begin{array}{l}\text { Small and } \\
\text { medium } \\
\text { enterprises }\end{array}$ & 254 & 4.21 & 4.31 & 4.35 & 4.44 & 4.58 & 4.72 & 4.51 & 4.45 & 4.67 \\
\hline
\end{tabular}


Table 14 shows that Chinese enterprises as a whole are slow in SINPD, but a later involvement does not necessarily mean a low involvement degree. The frequency of large enterprises' early-phase involvement and high involvement degree are higher than that of small and medium enterprises. However, their frequency of metaphase involvement and low involvement degree are lower than that of small and medium enterprises. Thus H1 is supported.

Table 15 Descriptive Statistics of SINPD Implementation in Different Typed Enterprises

\begin{tabular}{|c|c|c|c|c|c|c|c|c|c|c|}
\hline \multirow{2}{*}{ Enterprises type } & \multirow{2}{*}{ Obs. } & \multicolumn{6}{|c|}{ Involvement time } & \multicolumn{3}{|c|}{ Involvement degree } \\
\hline & & IVT1 & IVT2 & IVT3 & IVT4 & IVT5 & IVT6 & IVD1 & IVD2 & IVD3 \\
\hline $\begin{array}{l}\text { Domestic-funded } \\
\text { enterprises }\end{array}$ & 391 & 4.28 & 4.30 & 4.21 & 4.40 & 4.55 & 4.71 & 4.44 & 4.37 & 4.62 \\
\hline $\begin{array}{l}\text { Foreign-funded } \\
\text { enterprises }\end{array}$ & 38 & 4.61 & 4.63 & 4.58 & 4.63 & 4.77 & 4.71 & 4.56 & 4.48 & 4.77 \\
\hline
\end{tabular}

Table 15 shows that most domestic-funded enterprises and foreign-funded enterprises tend to engage in SINPD at metaphase and later stage of new product development, and demonstrate a high involvement degree. By comparison, the frequency of foreign-funded enterprises' early involvement and high involvement degree are higher than that of domestic-funded enterprises. Thus $\mathrm{H} 2$ is supported.

Table 16 Descriptive Statistics of SINPD Implementation in Enterprises in Different Industries

\begin{tabular}{|c|c|c|c|c|c|c|c|c|c|c|}
\hline \multirow{2}{*}{ Industry type } & \multirow{2}{*}{ Obs. } & \multicolumn{6}{|c|}{ Involvement time } & \multicolumn{3}{|c|}{ Involvement degree } \\
\hline & & IVT1 & IVT2 & IVT3 & IVT4 & IVT5 & IVT6 & IVD1 & IVD2 & IVD3 \\
\hline $\begin{array}{l}\text { Technology- } \\
\text { intensive } \\
\text { industries }\end{array}$ & 225 & 4.33 & 4.39 & 4.49 & 4.61 & 4.67 & 4.77 & 4.48 & 4.40 & 4.82 \\
\hline $\begin{array}{l}\text { Manufacturing } \\
\text { industries }\end{array}$ & 204 & 4.29 & 4.29 & 4.24 & 4.47 & 4.55 & 4.77 & 4.39 & 4.42 & 4.67 \\
\hline
\end{tabular}

Table 16 shows that the frequency of metaphase and later stage SINPD and high involvement degree in enterprises of technology-intensive industries are higher than that of enterprises in manufacturing industries. Thus, $\mathrm{H} 3$ is supported.

As above, the scores of SINPD in Chinese manufacturing enterprises mainly concentrate in the range of 4-5, indicating that SINPD in Chinese enterprises as a whole is still at an elementary stage. A lot has to be done to improve SINPD among Chinese enterprises. 
6.4 Relationship between Enterprise Characteristics and Driving Factors of SINPD

H4, H5 and H6 have been proposed to test the relationship between SINPD driving factors and enterprise characteristics (i.e., enterprise size, type and industry). Below are the multi-regression equations of the three hypotheses.

(1) Testing method: SINPD implementation as the dependent variable, six driving factors as the independent variables (performance expectation is further divided into short-term performance and long-term performance expectation). The equations of multi-regression analysis are shown as follows:

$\mathrm{SINPD}_{\text {large }}=0.087 \times$ external competition pressure $+0.205 \times$ national innovation policy guidance $+0.328 \times$ product complexity and technology integration $+0.173 \times$ R\&D dependence $+0.147 \times$ supplier dependence $+0.074 \times$ short-term performance expectation $+0.249 \times$ long-term performance expectation.

$\mathrm{SINPD}_{\text {small and medium }}=0.301 \times$ external competition pressures $+0.123 \times$ national innovation policy guidance $+0.185 \times$ product complexity and technology integration $+0.062 \times \mathrm{R} \& \mathrm{D}$ dependence $+0.189 \times$ supplier dependence $+0.109 \times$ short-term performance expectation $+0.209 \times$ long-term performance expectation.

In Equation (1), the values of the regression coefficients of national innovation policy guidance, product complexity, technology integration, R\&D dependence and long-term performance expectation are big, indicating that the implementation of SINPD in large enterprises are more likely to be driven by these 5 factors. Similarly, Equation (2) shows that the implementation of SINPD in small and medium enterprises is more likely to be driven by external competition pressures, supplier dependence and short-term performance expectation. Thus H4 is supported.

(2) Similarly, the multi-regression analysis equations of domestic-found and foreign-found enterprise are shown as follows, respectively.

$\mathrm{SINPD}_{\text {domestic-found }}=0.174 \times$ external competition pressure $+0.193 \times$ national innovation policy guidance $+0.256 \times$ product complexity and technology integration $+0.098 \times \mathrm{R} \& \mathrm{D}$ dependence $+0.011 \times$ supplier dependence $+0.130 \times$ short-term performance expectation $+0.275 \times$ long-term performance expectation.

$\mathrm{SINPD}_{\text {foreign-found }}=0.191 \times$ external competition pressure $+0.169 \times$ national innovation policy guidance $+0.368 \times$ product complexity and technology integration $+0.303 \times \mathrm{R} \& \mathrm{D}$ dependence $+0.191 \times$ supplier dependence $+0.053 \times$ short-term performance expectation $+0.121 \times$ long-term performance expectation....................................................... (4)

Equation (3) shows that the values of regression coefficient of national innovation policy guidance, expected short-term performance and expected 
long-term performance are big, indicating that the implementation of SINPD in domestic-found enterprises are more likely to be driven by these 3 factors. Likewise, Equation (4) shows that the implementation of SINPD in foreign-found enterprises is more likely to be driven by external competition pressures, product complexity, technology integration, R\&D dependence. H5 is supported.

(3) The multi-regression analysis equations for enterprises in technologyintensive manufacturing industries and other manufacturing industries are shown as follows:

$\mathrm{SINPD}_{\text {technology-intensive }}=0.250 \times$ external competition pressure $+0.049 \times$ national innovation policy guidance $+0.273 \times$ product complexity and technology integration $+0.082 \times \mathrm{R} \& \mathrm{D}$ dependence $+0.228 \times$ supplier dependence $+0.075 \times$ short-term performance expectation $+0.240 \times$ long-term performance expectation.............................................................

SINPD general $=0.079 \times$ external competition pressure $+0.213 \times$ national innovation policy guidance $+0.234 \times$ product complexity and technology integration $+0.171 \times \mathrm{R} \& \mathrm{D}$ dependence $+0.135 \times$ supplier dependence $+0.071 \times$ short-term performance expectation $+0.276 \times$ long-term performance expectation............................................................ (6)

Equation (5) shows that the implementation of SINPD in enterprises in technology-intensive industries are more likely to be driven by external competition pressure, product complexity and technology integration, and supplier dependence, while Equation (6) shows that national innovation policy guidance, short-term and long-term performance expectation are the main driving factors behind SINPD implementation in enterprises from non-technologyintensive industries. H6 is thus supported.

\subsection{Relationship between Enterprise Characteristics and Enabling Factors of} SINPD

With the regard to the influence of enterprise types on the enabling factors effect degree, $\mathrm{H} 7, \mathrm{H} 8$ and $\mathrm{H} 9$ have been proposed to test the relationship between SINPD enabling factors and enterprise characteristics (i.e., enterprise size, type and industry). Below are the multi-regression equations of the three hypotheses.

(1) SINPD as the dependent variable and four enabling factors as the independent variables. The multi-regression equations for large enterprises and small and medium enterprises are shown as follows, respectively.

$\mathrm{SINPD}_{\text {large }}=0.032 \times$ organization management $+0.120 \times$ human resources quality $+0.193 \times$ cooperation relationship $+0.350 \times$ mutual attraction .........(7)

$\mathrm{SINPD}_{\text {small and medium }}=0.236 \times$ organization management $+0.015 \times$ human resources quality $+0.181 \times$ cooperation relationship $+0.315 \times$ mutual attraction. 
The coefficient values of human resources quality, cooperation relationship and mutual attraction are rather big in Equation (7), showing that the implementation of SINPD in large enterprises are more likely to be driven by these factors. Likewise, Equation (8) shows that the implementation of SINPD in small and medium enterprises is more likely to be driven by organizational management. Thus $\mathrm{H} 7$ is supported.

(2) The multi-regression equations of domestic-found and foreign-found enterprises are shown as flows:

$\mathrm{SINPD}_{\text {domestic-found }}=0.119 \times$ organization management $+0.011 \times$ human resources quality $+0.238 \times$ cooperation relationship $+0.340 \times$ mutual attraction................................................................9.

$\mathrm{SINPD}_{\text {foreign-found }}=0.326 \times$ organization management $+0.422 \times$ human resources quality $+0.355 \times$ cooperation relationship $+0.508 \times$ mutual attraction

As shown, the implementation of SINPD in foreign-found enterprises is more likely to be driven by all the four factors in Equation 10: Hypothesis 8 is partially supported.

(3) The multi-regression analysis equations for enterprises in technologyintensive manufacturing industries and non-technology-intensive manufacturing industries are shown as follows:

$\mathrm{SINPD}_{\text {technology-intensive }}=0.015 \times$ organization management $+0.058 \times$ human resources quality $+0.281 \times$ cooperation relationship $+0.295 \times$ mutual attraction.

$\mathrm{SINPD}_{\text {general }}=0.319 \times$ organization management $+0.022 \times$ human resources quality $+0.145 \times$ cooperation relationship $+0.382 \times$ mutual attraction...

Equation (11) shows that the regression coefficient of are higher, indicates that the implementations of SINPD in enterprises in technology-intensive industries are more likely to driven by organizational management and mutual attraction, while Equation (12) also shows the implementation of SINPD in enterprises of non-technology-intensive industries are more likely to be driven by human resources quality and cooperation relationship. H9 is partially supported.

\section{Conclusion}

Based on meta-analysis of Western literature on the influencing factors of SINPD, and exploratory interview with Chinese manufacturing enterprises, we identify 12 driving factors and 12 enabling factors (in four different dimensions) for SINPD in China. After a large-sample empirical study, we extract 6 key driving factors for SINPD, namely external competition pressure, national innovation policy guidance, product complexity, technology integration, R\&D dependence, supplier dependence, and performance expectation. The four dimensions for 
SINPD enabling factors are named as follows: organizational management, human resource quality, cooperation relationship and mutual attraction. Specifically, the 12 enabling factors contained in these four dimensions are: organizational structure of the R\&D departments, new product development team, education and training level, previous experience of cooperation, perceived trust capabilities, technical expertise, mutual trust, information exchange systems, cultural compatibility, suppliers technical capabilities, manufacturer commitment, and geographic location.

We establish in this paper a model of influencing factors for SINPD. 9 hypotheses were proposed and tested. The results show that most of the SINPD in Chinese manufacturing enterprises occur at the middle or late stage of new product development. Suppliers enjoy a high involvement degree. In addition, there are significant differences in SINPD among different types of enterprises. However, in general, the status quo of SINPD in Chinese enterprises is not satisfactory and needs to be further developed.

We pay special attention to the impacts of different driving and enabling factors on SINPD implementation in different enterprises. The results show that, for enabling factors, the implementation of SINPD in large enterprises are mainly affected by human resources quality, cooperation relationship, and mutual attraction, while small and medium enterprises are mostly affected by organizational management. As for driving factors, our results show that all of them can affect foreign-funded enterprises. Except mutual attraction (while has a greater impact on foreign-funded enterprises than on domestic-funded enterprises), there are no significant differences in the impact of other driving factors on the two types of enterprises. We also find that the SINPD in technology-intensive manufacturing enterprises are more likely to be affected by organizational management and mutual attraction, while the SINPD in technology-intensive manufacturing enterprises are more likely to be affected by human resources quality and cooperation relationship.

This paper has important implications for Chinese enterprises' implementation of SINPD. First, manufacturing enterprises need to pay differentiated attention to different driving or enabling factors of SINPD to maximize supplier attribution in developing new products. Second, this paper contributes to development of SINPD study in China by providing empirical-study-based evidence. However, it also has several limitations. First, due to the limitations of both time and research budget, sampled enterprises were only from 10 provinces and there were fewer data from foreign-funded enterprises, which would impair the applicability of our conclusions to a certain degree. In addition, this article only focuses on the influencing factors of SINPD without taking into account the important issue of SINPD implementation. All these limitations should be the direction of future research. 


\section{References}

Anklesaria J, Burt D M (1987). Personal factors in the purchasing/engineering interface. Journal of Purchasing and Materials Management, 23: 9-18

Atuahene-Gima K (1995). Involving organizational buyers in new product development. Industrial Marketing Management, 24: 215-226

Bensaou M, Earl M J (2000). Corporate strategies: The right mindset for managing information technology, in: Garten G (ed.), World View. Cambridge, Mass: Harvard Business School Press

Bidault F, Despres C, Bulter C (1998). The drivers of cooperation between buyers and suppliers for product innovation. Research Policy, 26: 719-732

Birou L M, Fawcett S E (1994). Supplier involvement in integrated product development: A comparison of US and European practices. International Journal of Physical Distribution and Logistics Management, 24(5): 4-14

Bruce M, Leverick F, Littler D, Wilson D (1995). Success factors for collaborative product development: A study of suppliers of information and communication technology. R\&D Management, 25(1): 33-44

Burt D N, Soukup W R (1985). Purchasing's role in new product development. Harvard Business Review, 63(3): 90-97

Clark K B (1989). Project scope and project performance: The effect of parts strategy and supplier involvement on product development. Management Science, 35(10): 1247-1263

Clark K B, Fujimoto T (1991). Product development performance. Boston, MA: Harvard Business School Press

Dobler D W, Burt D N (1996). Purchasing and Materials Management: Text and Cases. New York: McGraw-Hill

Dowlatshahi S (1992). Purchasing's role in a concurrent engineering environment. International Journal of Operations and Production Management, 18(2): 143-167

Dowlatshahi S (1998). Implementing early supplier involvement: A conceptual framework. International Journal of Operations and Production Management, 18: 143-167

Duarte M, Davies G (2003). Testing the conflict performance assumption in business to business relationships. Industrial Marketing Management, 32: 91-99

Dyer J H, Ouchi W G (1993). Japanese-style partnerships: Giving companies a competitive edge. Sloan Management Review, 35(1): 51-63

Eisenhardt K M, Tabrizi B N (1995). Accelerating adaptive processes: Product innovation in the global computer industry. Administrative Science Quarterly, 40: 84-110

Farr C M, Fischer W A (1992). Managing international high technology co-operative projects. R\&D Management, 22(1): 77-95

Fliess S, Becker U (2006). Supplier integration-controlling of co-development processes. Industrial Marketing Management, 35: 28-44

Fujimoto T (1994). The origin and evolution of the black box parts practice in the Japanese auto industry. Paper presented at Fuji Conference, January, Japan

Gabarro J J (1987). The development of working relationships, in: Lorsch J W (ed.), Handbook of Organisational Behaviour. Englewood Cliffs, NJ: Prentice Hall, Inc.

Griffin A, Page A (1996). PDMA success measurement project: Recommended measures for product development success and failure. Journal of Product Innovation Management, 13: 478-496

Guy S P, Dale B G (1993). The role of purchasing in design: A study in the British defense 
industry. International Journal of Purchasing and Materials Management, 29(3): 27-31

Handfield R B, Ragatz G L, Petersen K J, Monczka R M (1999). Involving suppliers in new product development. California Management Review, 42(1): 59-82

Hartley J L, Zirger B J, Kamath R R (1997). Managing the buyer-supplier interface for on-time performance in product development. Journal of Operations Management, 15: 57-70

Hoegl M, Wagner S M (2005). Buyer-supplier collaboration in product development projects. Journal of Management, 31(4): 530-548

Imai K, Nonaka I, Takeuchi H (1985). Managing the new product development process: How Japanese companies learn and unlearn, in: Clark K B, Hayes R H, Lorenz C (eds.), The Uneasy Alliance: Managing the Productivity-Technology Dilemma. Boston, MA: Harvard Business School Press, 337-375

Kaiser H F (1974). An index of factorial simplicity. Pschometrika, 39: 31-6

Lamming R (1993). Beyond Partnership: Strategies for Innovation and Lean Supply. UK: Prentice-Hall International Ltd.

Littler D, Leverick F, Bruce M (1995). Factors affecting the process of collaborative product development: A study of UK manufacturers of information and communications technology products. Journal of Product Innovation Management, 12: 16-32

Lorange P (1988). Co-operative strategies: Planning and control considerations, in: Hood N, Vahlne J E (eds.), Strategies in Global Competition. London: Croom Helm, 370-389

McIvor R, Humphreys P (2004). Early supplier involvement in the design process: Lesson from the electronics industry. The International Journal of Management Science, 32: 179-199

Perlmutter H V, Heenan D A (1986). Co-operate to compete globally. Harvard Business Review, 64(2): 136-152

Petersen K J, Handfield R B, Ragatz G L (2003). A model of supplier integration into new product development. Journal product innovation management, 20: 284-299

Petersen K J, Handfield R B, Ragatz G L (2005). Supplier integration into new product development: Coordinating product, process and supply chain design. Journal of Operations Management, 23: 371-388

Ragatz G L, Handheld R B, Scannell T V (1997). Success factors for integrating suppliers into new product development. Journal Product Innovation Management, 14: 190-202

Sako M (1992). Prices, Quality and Trust: Inter-Firm Relations in Britain and Japan. Cambridge: Cambridge University Press

Song X M, Parry M E (1999). Challenges of managing the development of breakthrough products in Japan. Journal of Operations Management, 17: 665-688

Tatikonda M V, Rosenthal S R (2000). Technology novelty, project complexity, and product development project execution success: A deeper look at task uncertainty in product innovation. IEEE Transactions on Engineering Management, 47(1): 74-87

Van Echtelt F E A, Wynstra F (2001). Managing supplier integration into product development: A literature review and conceptual model, in: Verspagen B (ed.), Proceedings $17^{\text {th }}$ ECIS Conference, The Future of Innovation Studies. Eindhoven University of Technology, The Netherlands

Von Hippel E (1990). Task partitioning: An innovation process variable. Research Policy, 19: 407-418

Wagner S M, Hoegl M (2006). Involving supplier in product development: Insights from R\&D directors and project managers. Industrial Marketing Management, 35: 936-943

Walter A (2003). Relationship-specific factors influencing supplier involvement in customer new product development. Journal of Business Research, 56: 721-733 
Wasti S N, Liker J K (1997). Risky business or competitive power? Supplier involvement in Japanese product design. Journal of Product Innovation Management, 14: 337-355

Whipple J M, Frankel R (2000). Strategic alliance success factors. Journal of Supply Chain Management, 8: 21-28

Wynstra F, Axelsson B, Van Weele A J (2000). Driving and enabling factors for purchasing involvement in productdevelopment. European Journal of Purchasing and Supply Management, 6(2): 129-141

Wynstra F, Pierick T (2000). Managing supplier involvement in product development: A portfolio approach. European Journal of Purchasing and Supply Management, 6: 49-57

Wynstra F, Weggeman M, Van Weele A J (2003). Exploring purchasing integration in product development. Industrial Marketing Management, 32(1): 69-83 